\title{
Diffusion-driven nucleation from surface nuclei in hydrodynamic cavitation
}

\author{
T. F. Groß ${ }^{1}$ and P. F. Pelz ${ }^{1, \dagger}$ \\ ${ }^{1}$ Chair of Fluid Systems, Technische Universität Darmstadt, Otto-Berndt Straße 2, \\ 64287 Darmstadt, DE, Germany
}

(Received 10 February 2017; revised 20 July 2017; accepted 15 August 2017; first published online 29 September 2017)

Investigations about the role of nuclei and nucleation for the inception and formation of cavitation have been part of cavitation research since Harvey et al. (J. Cell. Physiol., vol. 24 (1), 1944, pp. 1-22) postulated the existence of gas filled crevices on surfaces and particles in liquids. In a supersaturated liquid, surface nuclei produce small gas bubbles due to mass transfer of gas or themselves work as weak spots in the liquid that are necessary for a phase change under technically relevant static pressures. Although various theories and models about nuclei and nucleation have found their way into standard literature, there is a lack of experimentally validated theories that describe the process of diffusion-driven nucleation in hydrodynamic cavitation. In order to close this gap we give new theoretical insights into the physics of this nucleation mechanism at technically relevant low supersaturations validated with extensive experimental results. The nucleation rate, the number of produced bubbles per second, is proportional to the supersaturation of the liquid and shows a nonlinear dependence on the shear rate at the surface nucleus. A model for the Strouhal number as dimensionless nucleation rate is derived allowing the estimation of nucleation rates from surface nuclei in hydrodynamic cavitation. The model provides three asymptotes, being a function of Péclet number, Weber number, the supersaturation of the liquid $\zeta$ and gas solubility $\Lambda$ for three different detachment mechanisms, $S r \propto \zeta \Lambda W e^{n} P e^{1 / 3}$ with $n=1 / 3,3 / 4,1$. The theoretical findings are in good agreement with experimental results, leading to a new assessment of the role of diffusion in cavitating flows.

Key words: cavitation, drops and bubbles, multiphase flow

\section{Introduction}

The occurrence of cavitation in hydraulic machines and maritime applications is usually accompanied by negative aspects. Cavitation in pumps, turbines and nozzles, as well as on propellers and rudders, influences the characteristic curves, is responsible for noise and vibration, and causes damage of structural elements which is called cavitation erosion (Brennen 1995; Pelz, Keil \& Ludwig 2014). Furthermore, cavitation is a research topic because of its useful application in life science, medicine and process engineering. The cleaning of surfaces (Verhaagen \& Fernandez Rivas 2015),

$\dagger$ Email address for correspondence: peter.pelz@fst.tu-darmstadt.de 
the removal of bacteria and germs in water (Sarc, Oder \& Dular 2016), the fragmentation of kidney stones in the human body by acoustic cavitation called lithotripsy, the targeted release of pharmacological agents (both Brennen 2015), and the enhancement of the efficiency of mixing processes (Spiridonov 2015) are only examples for the far-reaching application of hydrodynamic and acoustic cavitation. In the present paper the focus is on hydrodynamic cavitation. The presented findings can also be applied to other research fields in which bubble formation is of importance, e.g. oil hydraulic devices, microfluidic devices or biofluidic systems.

Hydrodynamic cavitation occurs in diverse appearances - the so-called cavitation regimes. As examples, the terms single bubble cavitation, streak cavitation, tip vortex cavitation, sheet cavitation and cloud cavitation describe the macroscopic appearance of cavitating flows. Regardless of the cavitation regime, there is one fundamental similarity: the smallest elements of cavitating flows are cavitation nuclei. Without an understanding of nucleation the picture of cavitation is incomplete. To motivate our research there is a need to review the existent nuclei and nucleation theories shown in figure 1. We distinguish between equilibrium and non-equilibrium theories with respect to the solved gas content, i.e. supersaturation of the liquid. The supersaturation of the liquid $\zeta$ is the relevant quantity that allows the quantification of gas being solved in the liquid in relation to the equilibrium state and is important to describe diffusion processes. $\zeta=0$ is the equilibrium state. The definition of the supersaturation is available later in (2.3). In cavitation research the focus is mainly on equilibrium processes, whereas experiments indicate that non-equilibrium processes, i.e. diffusion processes, are relevant as well (Pelz, Keil \& Groß 2017).

\subsection{General assessment of nuclei and nucleation theories}

Basically, cavitation nuclei are small amounts of non-condensable gas that work as weak spots in the liquid and allow its rupture under technical relevant pressures. The well-known experiments of Briggs (1950) demonstrate the ability of liquids to withstand high tensile stresses if the presence of such nuclei is avoided, e.g. a tensile strength of $28 \mathrm{MPa}$ in water at $10^{\circ} \mathrm{C}$. Theoretical considerations, for example, based on the Van der Waals equation (Benson \& Gerjuoy 1949), suggest that the tensile strength is of the order of $100 \mathrm{MPa}$ if the presence of nuclei is avoided. Mørch (2007) gives a comprehensive overview of studies in which the dependence of the tensile strength on the nuclei concentration are investigated. In technical flows one only observes small tensile strengths, which leads to the conclusion that there is always a large number of nuclei existing in the liquid.

In cavitation research a distinction is made between bubbles and particles that freely float in the liquid and nuclei that are attached to walls, see figure 1. Bubbles and particles in the bulk of the liquid are referred to as free-stream nuclei while nuclei present in the walls bounding the flow are referred to as surface nuclei (Brennen 1995). In their works on bubble formation in animals, Harvey et al. (1944) postulated the existence of minute gas nuclei attached to hydrophobic cracks in surfaces and particles. A widespread opinion is that the onset of cavitation is mainly associated with free-stream nuclei, and surface nuclei only play a minor role (cf. $\$ 1.11$ of Brennen (1995)). This statement is true for some cavitation regimes (tip vortex cavitation, single bubble cavitation), but indeed not generally valid for all cavitation regimes, as our own experiments indicate (Pelz et al. 2017) and as we will see in the following.

It is a known fact that the number density of free-stream nuclei, i.e. micro-bubbles and particles, has to be considered as well as their long-term stability. In order to 


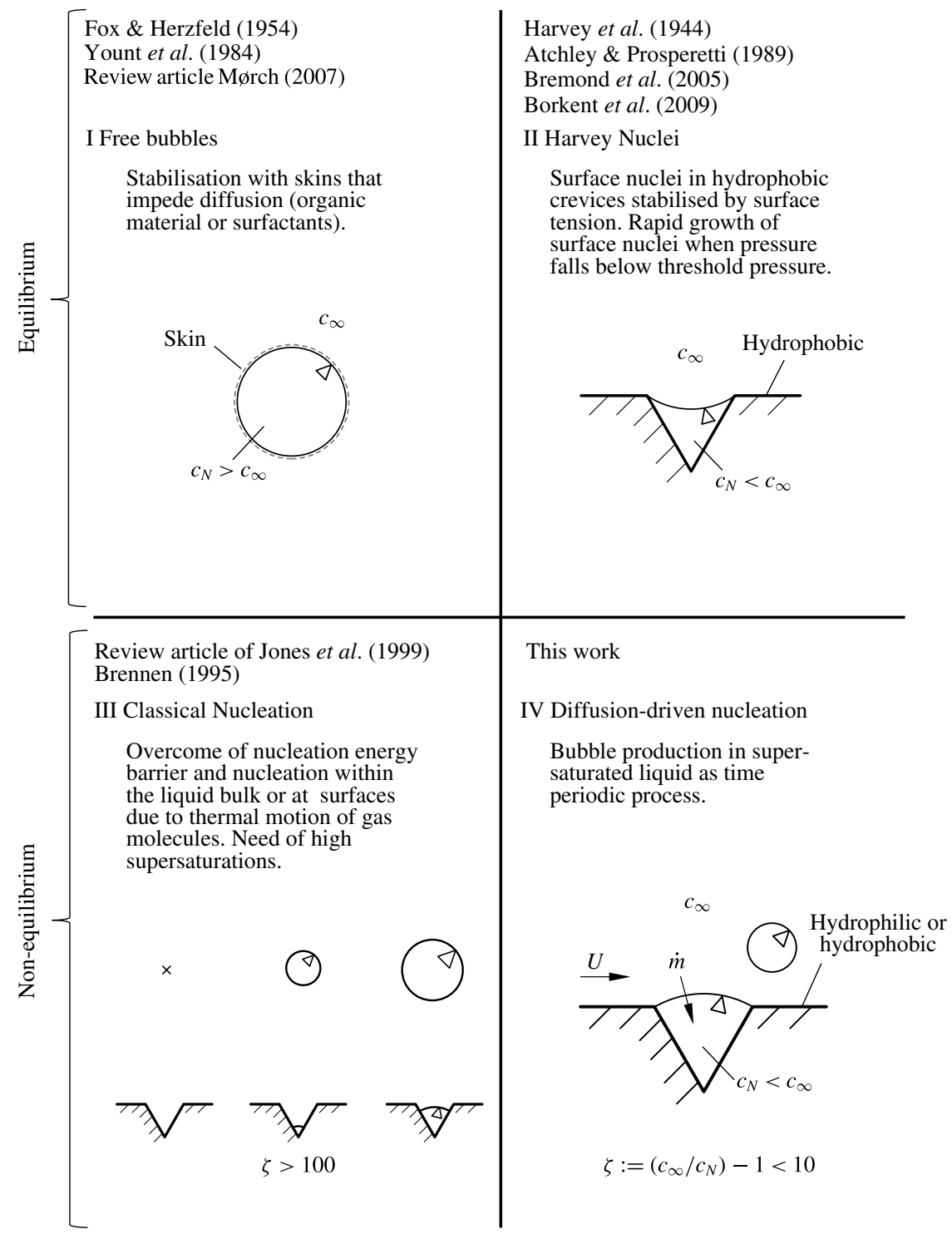

FIGURE 1. Schematic overview of nuclei and nucleation in liquids. Equilibrium and nonequilibrium theories with respect to the solved gas content (supersaturation of the liquid) are distinguished.

serve as cavitation nuclei permanently, both free-stream nuclei and surface nuclei must be stabilised in some way. Dispersed bubbles are unstable since they grow or shrink due to diffusion caused by the effect of surface tension. They are supposed to be stabilised by skins that impede the diffusion of gas, and therefore limit growth and shrinking, resulting in an equilibrium, cf. figure 1 I. Fox \& Herzfeld (1954) developed a theory of an organic skin while Yount's theory implies the existence of a skin of surface-active substances (Yount, Gillary \& Hoffman 1984). The stabilisation of nuclei in hydrophobic surfaces, the so-called Harvey nuclei, can be explained 
by the effect of surface tension as well (Harvey et al. 1944; Atchley \& Prosperetti 1989), cf. figure 1 II: since the surface tension is acting on the convex curved liquid-gas interface the pressure in the gas pockets is lower than the pressure in the surrounding liquid. Because of the lower pressure the surface nuclei do not dissolve in the surrounding liquid, unless it is strongly undersaturated. Nuclei on hydrophilic surfaces are supposed to be unstable unless they are also stabilised by skins. It is often assumed that the liquid is in a concentration equilibrium which usually applies for quiescent liquids, e.g. in acoustic cavitation applications, but does not apply for the low-pressure region in hydrodynamic cavitation where the liquid is supersaturated.

The theories of free-stream nuclei and surface nuclei have been well established and can be found in the standard literature (e.g. Knapp, Daily \& Hammitt 1970; Franc \& Michel 2004). Recent studies about nuclei on hydrophobic surfaces (Bremond et al. 2005; Borkent et al. 2009), skin-stabilised surface nuclei (Andersen \& Mørch 2015), the activation of roughness elements that serve as nucleation spots (van Rijsbergen \& van Terwisga 2011), vapour-bubble nucleation in Rayleigh-Bénard turbulence (Guzman et al. 2016), vapour bubbles and the role of non-condensable gas (Prosperetti 2017), and the broad field of nano-bubbles (Lohse \& Zhang 2015) underline the importance of research on cavitation nuclei as well as on (micro- and nano-) bubbles.

In contrast to the stabilisation theories, the process of bubble nucleation is investigated much less in the context of hydrodynamic cavitation. In cavitation research the term nucleation is used to denote the formation of a bubble that works as a weak spot in the liquid and allows its rupture. Research on nucleation processes are important for the understanding of the occurrence of the diverse cavitation regimes and cavitation inception, especially regarding the origin of bubbles serving as cavitation nuclei (Pelz et al. 2017). Usually a differentiation is made between bubble formation within the liquid bulk (homogeneous nucleation) and bubble formation at weak spots in the liquid, i.e. gas bubbles, pre-existing gas cavities, surfaces, particles (heterogeneous nucleation).

In cavitation research the terms homogeneous nucleation and the classical nucleation theory are used synonymously. The classical nucleation theory describes the formation of bubbles within the liquid bulk due to thermal motion of gas molecules, cf. figure 1 III. It is reasonable to assume that homogeneous nucleation requires high levels of supersaturation, i.e. $\zeta>100$, because it is necessary to overcome the tensile strength of the liquid to create the new phase, being known as the nucleation energy barrier. It is common sense that homogeneous nucleation is not relevant for hydrodynamic cavitation.

In cavitation research it is widely accepted that nucleation is triggered by the growth of minute gas bubbles which are entrapped in crevices (surface nuclei) when the pressure falls below a critical threshold pressure. This process is called heterogeneous nucleation. The crevice model of bubble nucleation of Atchley \& Prosperetti (1989) contains both the initial growth of the interface in the crevice and the process by which the interface moves out of the crevice. The theory has been validated by Bremond et al. (2005) and is of particular importance for acoustic cavitation. Even though the crevice model is widespread it is uncertain which role it plays in hydrodynamic cavitation, since gas diffusion is neglected. Even Atchley \& Prosperetti (1989) stated that, in hydrodynamic cavitation, gas diffusion is of importance and a different nucleation behaviour would be expected.

In the present paper the focus is on a nucleation process where gas diffusion is the driving mechanism. Surface nuclei grow due to gas diffusion when a supersaturated 
liquid flows over the surface where the nuclei are located, cf. figure $1 \mathrm{IV}$. When a surface nucleus reaches a critical size a part of it detaches as a bubble. This process repeats so that a periodical production of bubbles can be observed. Depending on their size, the produced bubbles may then act as cavitation nuclei when entering a low pressure region, cf. Blake's critical radius Blake (1949). We call this process diffusion-driven nucleation to relate it to the other mentioned nucleation theories in cavitation research. The number of produced bubbles per second is called the nucleation rate. Indeed, the terms bubble formation and bubble formation rate are also correct descriptions of the process. As we will see in the following, the bubbles produced at surface nuclei by diffusion-driven nucleation have a great impact on cavitating flows.

\subsection{Diffusion-driven nucleation in quiescent liquids and fluid flows}

When speaking about diffusion in the context of cavitation, usually the work of Epstein \& Plesset (1950) is cited. In their work the authors investigate the time a gas bubble needs for the dissolution in an undersaturated liquid as well as the time needed to increase its radius by tenfold in a supersaturated liquid. Neglecting relative translational motion of the bubble, a typical time for the solution of a bubble with an initial radius of $10 \mu \mathrm{m}$ is $2.5 \mathrm{~s}$. This finding is often used to argue that diffusion processes (and thus diffusion-driven nucleation) cannot be of importance for cavitating flows since typical times, usually the bubble collapse time, are much shorter (Rayleigh collapse time $\tau \sim 1 \mu \mathrm{s}-10 \mu \mathrm{s}$, see Franc \& Michel (2004)). For the bubble dynamics this conclusion is valid and diffusion only plays, if any, a minor role. In the case of diffusion-driven nucleation from surface nuclei this argument misses the point because the processes, i.e. nucleation and cavitation, are on different time scales. In addition, Epstein \& Plesset (1950) studied the growth and shrinkage of bubbles in a quiescent liquid. Parkin \& Kermeen (1963) and van Wijngaarden (1967) investigated the influence of convection on the diffusion mass flux, and thus on the growth of small bubbles. As one expects, a forced convection due to a fluid flow intensifies the diffusion mass flux, and thus leads to larger growth rates of the bubbles.

Only a few investigations allow a closer and direct look at diffusion-driven nucleation from surface nuclei in hydrodynamic cavitation. Thus, it is worthwhile to study the relevant literature on (diffusion-driven) bubble formation. Besides others, Liger-Belair (2005) examines bubble formation in carbonated beverages. In a glass of champagne, cellulose fibres with a diameter of $10 \mu \mathrm{m}-20 \mu \mathrm{m}$, and thus not visible to the naked eye, work as nucleation sites. The observed nucleation rates, i.e. number of produced bubbles per second, depend on the size of the fibres so that different frequencies can be observed at the same time at fibres of different sizes. Since the nucleation rate depends on the supersaturation of the liquid, it decreases with time as the champagne becomes stale and flat. These results appear to be unsuitable for technically relevant issues at first glance, but even in this quiescent environment nucleation rates up to $30 \mathrm{~Hz}$ are observable (Liger-Belair 2005). One has to keep in mind that the solubility of water for carbon dioxide is approximately 50 times higher than its solubility for air. Besides others, the contributions of Lubetkin (1989) and Jones et al. (1997) are also important works on this topic. Lubetkin (1994) and Jones, Evans \& Galvin (1999) provide a good overview of relevant works on bubble formation. Important to mention is the work of Bankoff (1958), who presented theoretical considerations about the entrapment mechanism of gas on rough surfaces. These gas entrapments work as nucleation sites. 
(a)

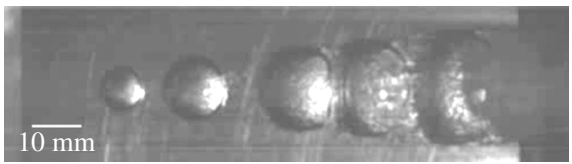

Single picture (b)

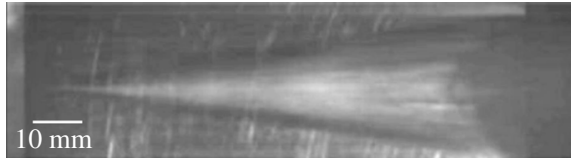

Averaged picture

FIGURE 2. Streak cavitation on the NACA 0009 hydrofoil. The flow is from left to right with a frequency of bubble generation of $1000 \mathrm{~Hz}$. (a) Picture taken with an exposure time of $55 \mu \mathrm{s}$. (b) Averaged picture of 50 single images. The cavitation number is 5.4 and the Reynolds number is $1.1 \times 10^{5}$. The image was taken at the cavitation tunnel of the Laboratory for Hydraulic Machines/École Polytechnique Fédérale de Lausanne by the authors. Also see Guennoun et al. (2003), Guennoun (2006) and Pelz et al. (2017).

The previously mentioned studies about bubble formation have in common that the process is studied in a quiescent liquid. It is to be expected that a fluid flow affects the studied nucleation process, i.e. the growth of a surface nucleus and the detachment of bubbles. A more appropriate flow condition is the flow around a hydrofoil. Guennoun et al. (2003), Guennoun (2006) observed nucleation from surface nuclei and measured nucleation rates of approximately $5 \mathrm{kHz}$ on a NACA 0009 hydrofoil, cf. figure 2(a). For the naked eye those bubbles create the appearance of a conical streak, figure $2(b)$. One immediately recognises that the origin of the streak is a specific point on the surface of the observed object. In the shown case the point of origin is a drilled blind hole on the hydrofoil in which a pressure transducer is located (cf. Guennoun et al. 2003; Guennoun 2006). Because of its temporal and spatial regularity this case serves as a good example for the theoretical considerations presented in the following. Guennoun et al. have not considered diffusion-driven nucleation as a cause for the cyclic production of bubbles. In $\S 5$ we will pick this case up again to prove the practical applicability of the approach presented in this paper.

Peters \& Honza (2014) systematically studied diffusion-driven nucleation from surface nuclei in silicone oil using a laminar radial gap flow. Blind holes with diameters of $0.6 \mathrm{~mm}$ and $0.8 \mathrm{~mm}$ served as nucleation sites. The investigated flow velocities were relatively small $\left(\sim 0.1 \mathrm{~m} \mathrm{~s}^{-1}\right.$ and smaller $)$. The authors noticed that the nucleation rate depends on the shear stress at the wall. Due to a narrow gap, shear rates of the order of magnitude $10^{2} \mathrm{~s}^{-1}-10^{3} \mathrm{~s}^{-1}$ were realised, resulting in nucleation rates of the order of magnitude $0.01 \mathrm{~Hz}-10 \mathrm{~Hz}$. Groß, Ludwig \& Pelz (2016) improved the experimental set-up used by Peters \& Honza and increased the flow velocities up to $2 \mathrm{~m} \mathrm{~s}^{-1}$ and shear rates of the order of magnitude of $1 \times 10^{4} \mathrm{~s}^{-1}$. Furthermore, it was possible to vary flow velocity and supersaturation separately. The authors observed nucleation rates of the order of $0.1 \mathrm{~Hz}-1000 \mathrm{~Hz}$ and found that there is a linear dependence of the nucleation rate on the supersaturation and a nonlinear dependence on the wall shear rate. The experiments were also carried out with silicone oil.

The study of the existing literature and current research activities shows that there is a lack of experiments and validated theoretical investigations that describe the process of diffusion-driven nucleation from surface nuclei in the context of hydrodynamic cavitation. In order to close this gap we used the generic experiment designed for silicone oil presented in Groß et al. (2016) and modified it to execute experiments with water. The experimental findings support an extended understanding of diffusiondriven nucleation as a self-excited cyclic process and help us to develop physical models. 

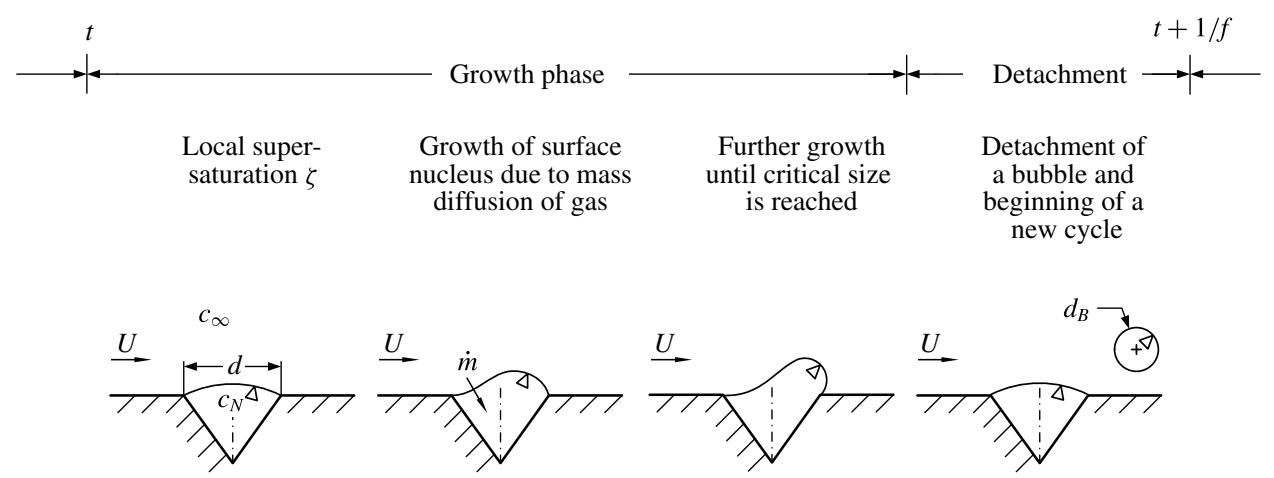

FIGURE 3. Principle sketch of the growth of a surface nucleus and the detachment of a bubble in a fluid flow.

The paper is outlined as follows. In $\S 2$ we provide theoretical considerations of the described nucleation process, including the growth of surface nuclei and the detachment of bubbles. The experimental set-up and the experimental procedure are explained in $\S 3$. In $\S 4$ the experimental results are presented and compared with the theoretical findings. In $\S 5$ the results are applied to cases of technical relevance. The paper closes with a conclusion in $\S 6$.

\section{Cycle frequency of diffusion-driven nucleation}

We consider a crevice that is filled with non-condensable gas surrounded by a flowing, supersaturated liquid, as sketched in figure 3. Due to the supersaturation of the liquid there is a mass flux of gas out of the liquid into the surface nucleus. According to Fick's law (Fick 1855), the mass flux of gas that diffuses into the surface nucleus, $\dot{m}$, is proportional to the concentration gradient at the liquid-gas interface,

$$
\dot{m}=-M \mathcal{D} \int_{A} \nabla c \cdot \boldsymbol{n} \mathrm{d} A,
$$

with concentration $c$ measured in mol $\mathrm{m}^{-3}$, diffusion coefficient $\mathcal{D}$, molar mass $M$, surface area of the nucleus $A$ and the surface normal vector $\boldsymbol{n}$. In an equilibrium the concentration of gas that is solved in the liquid increases linearly with the pressure, as described by Henry's law (Henry 1803),

$$
c=p \mathcal{H}
$$

with pressure $p$ and Henry coefficient $\mathcal{H}$ accordingly measured in mol $\left(\mathrm{m}^{3} \mathrm{~Pa}\right)^{-1}$. Neglecting pressure differences due to surface tension, a surface nucleus that is surrounded by a liquid in a concentration equilibrium will not show diffusion-driven nucleation since the concentration gradient is zero. Taking surface tension into account leads to the stability discussion referred to above.

In the case of a pressure decrease the liquid becomes supersaturated. The supersaturation is defined as

$$
\zeta:=\frac{c_{\infty}}{c_{N}}-1
$$

$c_{\infty}$ is the concentration of gas in the liquid and $c_{N}=p_{N} \mathcal{H}$ is the local saturation concentration at the surface nucleus, which is determined by the partial pressure 


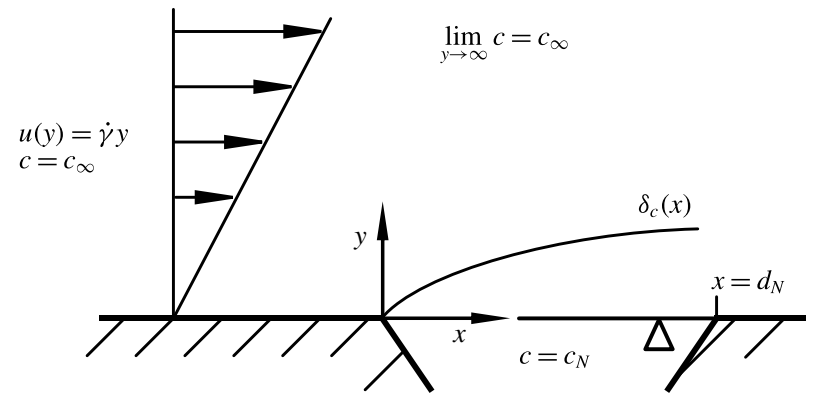

FIGURE 4. Sketch of the advection-diffusion problem at a surface nucleus. Evolution of a concentration field with concentration boundary layer thickness $\delta_{c}$.

of non-condensable gas inside the surface nucleus $p_{N}$. A concentration distribution develops.

The surface nucleus grows and when reaching a critical size a part of the surface nucleus detaches as a bubble with mass $m_{B}$ and diameter $d_{B}$, see figure 3 . Depending on its size, this bubble might serve as cavitation nucleus when carried into the lowpressure region of the flow, cf. Blake (1949). A specific amount of gas, approximately the same for every event, remains in the surface nucleus and the process repeats. From daily life we know that this process will last as long as the liquid is supersaturated. In technical flows where cavitation (or degassing) can be observed there is always 'new' liquid that flows past the surface nucleus. This keeps the process alive for an arbitrarily long time since the average saturation concentration in the system is higher than the saturation concentration in the low-pressure region. Hence, the liquid in the low-pressure region is supersaturated. Active surface nuclei, i.e. surface nuclei that produce bubbles, are an inexhaustible source for bubbles that might act as cavitation nuclei. Then, stabilisation theories of free-stream nuclei (Fox \& Herzfeld 1954; Yount et al. 1984) fade into the background.

The growth of the surface nucleus is relatively slow while the bubble detachment occurs rapidly. The nucleation rate $f$, the number of produced bubbles per second (see figure 3), can therefore be calculated with

$$
f=\frac{\dot{m}}{m_{B}} .
$$

This relation will guide us in the following and helps us to derive a physical model that describes the nucleation process based on axiomatic equations. The mass flux $\dot{m}$ is treated in $\S 2.1$ and the mass of the detaching bubbles $m_{B}$ in $\S 2.2$. In our analysis we execute an elementary discussion rather than a full mathematical and physical treatment of the problem. The focus of the paper is to identify the main influencing variables and to draw general conclusions about diffusion-driven nucleation.

\subsection{Growth phase of the nucleus}

The mass flux $\dot{m}$ that diffuses into a surface nucleus can be obtained by formulating a plane generic problem, see figure 4. As already mentioned, the concentration gradient at the liquid-gas interface is needed to calculate the mass flux. Solving the diffusion problem for the growth phase will guide as to $S h \approx 0.66 P \mathrm{e}^{1 / 3}$ for the dimensionless mass flux of gas that diffuses into the surface nucleus (definitions follow in (2.6)). 
In the most general case, the concentration field in the liquid satisfies the transient advection-diffusion equation

$$
\frac{\partial c}{\partial t}+\boldsymbol{u} \cdot \nabla c=\nabla \cdot(\mathcal{D} \nabla c),
$$

where $\boldsymbol{u}$ is the velocity field of the fluid flow. We assume a stationary process, a negligible flow in the $y$ direction, negligible diffusion in the $x$ direction and a constant diffusion coefficient $\mathcal{D}=$ const. Near the solid surface the velocity increases linearly with the wall distance $u=\dot{\gamma} y$ as long as the crevices are much smaller than the typical length of the device (diameter of a pipe, height of a gap). The velocity profile $u=\dot{\gamma} y$ is also valid in turbulent flows if the typical size of the crevice $d$ is smaller than the viscous length $l_{v}:=v / \sqrt{\tau_{w} / \varrho}$, with viscosity $v$ and density $\varrho$ of the fluid and wall shear stress $\tau_{w}$. The former approach is called the Lévêque approximation and is a common practice for solving heat and mass transfer problems, e.g. the asymmetric Graetz problem (Edwards \& Newman 1985). If $\dot{\gamma}$ is the relevant kinematic quantity near the solid wall and near the crevice, the suitable definitions of Sherwood number and Péclet number are

$$
S h:=\frac{\dot{m}}{c_{N} \zeta M \mathcal{D} d}, \quad P e:=\frac{\dot{\gamma} d^{2}}{\mathcal{D}} .
$$

The length $d$ denotes the diameter of a circular crevice having the same surface area as a hypothetical rectangular nucleus with side length $d_{N}=d \sqrt{\pi} / 2$. For $P e \gg 1$ the boundary layer approximation (Schlichting \& Gersten 2006) applied to (2.5) gives

$$
\dot{\gamma} y \frac{\partial c}{\partial x}=\mathcal{D} \frac{\partial^{2} c}{\partial y^{2}},
$$

with the boundary conditions $c(x=0, y)=c(x, y \rightarrow \infty)=c_{\infty}$ and $c\left(0<x<d_{N}, y=0\right)=$ $c_{N}$. Using $c_{+}:=\left(c-c_{N}\right) /\left(c_{\infty}-c_{N}\right), x_{+}:=x \mathcal{D} /\left(\dot{\gamma} d_{N}^{3}\right), d_{N+}:=\mathcal{D} /\left(\dot{\gamma} d_{N}^{2}\right)$ and $y_{+}:=y / d_{N}$ yields the dimensionless formulation

$$
y_{+} \frac{\partial c_{+}}{\partial x_{+}}=\frac{\partial^{2} c_{+}}{\partial y_{+}^{2}},
$$

with $c_{+}\left(x_{+}=0, y_{+}\right)=c_{+}\left(x_{+}, y_{+} \rightarrow \infty\right)=1$ and $c_{+}\left(0<x_{+}<d_{N+}, y_{+}=0\right)=0$. With the similarity variable $\eta:=y_{+} / x_{+}^{1 / 3}$, i.e. $c_{+}=c_{+}(\eta)$, the boundary value problem is equivalent to

$$
\frac{\mathrm{d}^{2} c_{+}}{\mathrm{d} \eta^{2}}+\frac{1}{3} \eta^{2} \frac{\mathrm{d} c_{+}}{\mathrm{d} \eta}=0,
$$

with $c_{+}(\eta=0)=0$ and $c_{+}(\eta \rightarrow \infty)=1$. The transformation $\mu:=\eta^{3} / 9$ yields the solution

$$
c_{+}(\mu)=\frac{\Gamma(1 / 3)-\Gamma(1 / 3, \mu)}{\Gamma(1 / 3)} .
$$

The series expansion of the incomplete Gamma function $\Gamma(1 / 3, \mu)$ gives the expanded solution

$$
c_{+}(\mu)=\frac{3 \mu^{1 / 3}-3 / 4 \mu^{4 / 3}+O\left(\mu^{7 / 3}\right)}{\Gamma(1 / 3)} .
$$


Considering only the leading term results in the approximate solution for the region near to the surface nucleus,

$$
\frac{c(x, y)}{c_{N}} \approx 1+\zeta \frac{3^{1 / 3}}{\Gamma(1 / 3)}\left(\frac{\dot{\gamma} d_{N}^{2}}{\mathcal{D}}\right)^{1 / 3}\left(\frac{x}{d_{N}}\right)^{-1 / 3} \frac{y}{d_{N}} .
$$

As a result, for $u=\dot{\gamma} y$, the concentration field and the mass flux $\dot{m}$ are proportional to $P e^{1 / 3}$. The latter is given by the surface integral

$$
\dot{m}=\left.\mathcal{D} M d_{N} \int_{0}^{d_{N}} \frac{\partial c}{\partial y}\right|_{y=0} \mathrm{~d} x \approx \mathcal{D} M d \zeta c_{N} \frac{3^{4 / 3} \pi^{5 / 6}}{2^{8 / 3} \Gamma(1 / 3)} P e^{1 / 3}
$$

or dimensionless

$$
S h \approx 0.66 P e^{1 / 3} .
$$

In our derivation we assume the no-slip condition at the solid wall and expect the velocity field to be unaffected when flowing over the surface nucleus. This does not imply the no-slip condition at the liquid-gas interface, cf. §2.2. If the flow is better described by a uniform flow profile, $u(y)=U_{\infty}$, one gets the solution $S h=\pi^{1 / 4} 2^{-1 / 2}\left(U_{\infty} \mathrm{d} / \mathcal{D}\right)^{1 / 2} \approx 0.94 P e^{\prime / 2}$ with the velocity of the undisturbed flow $U_{\infty}$ (Higbie 1935). This expression is an upper bound for the mass flux. Parkin \& Kermeen (1963) considered the growth of gas bubbles in the boundary layer of a submerged body due to convective air diffusion and get a similar solution. They additionally take diffusion in the flow direction into account. The work of Parkin and Kermeen is one of the few works that consider the intensification of the diffusion mass flux due to convective diffusion in the context of hydrodynamic cavitation.

The results $S h \approx 0.66 P e^{1 / 3}$ and $S h \approx 0.94 P e^{\prime 1 / 2}$ can be compared to the academic case studied by Epstein \& Plesset (1950) mentioned before. The steady state solution for the mass flux that diffuses into a resting spherical bubble with surface area $\pi d^{2} / 4$ is $S h=\pi$. A comparison of the three solutions shows that advection of course intensifies the mass flux of gas into the surface nucleus. It may be observed that the constant value for the steady case can be larger than the results of the convective approaches in the case of small Péclet numbers. The reason is that the previous assumption of negligible diffusion in the $x$ direction is only valid for $P e \gg 1$ or $P e^{\prime} \gg 1$, respectively. Thus, a comparison of the three cases only makes sense for large Péclet numbers, which usually is fulfilled in cavitating flows.

In the following we use the derived relation $S h \propto P e^{1 / 3}$, which implies $\dot{m} \propto \dot{\gamma}^{1 / 3}$ in dimensional quantities. Following equation (2.4), both factors $\dot{m}$ and $1 / m_{B}$ determine the nucleation rate. As will be seen later, $1 / m_{B}$ also follows a power law with the shear rate, showing an exponent even greater than $1 / 3$. Thus, the influence of the shear rate on the mass of the bubbles is greater than its influence on the mass flux. An upper bound for the nucleation rate can be determined by using the relation $S h \propto P e^{1 / 2}$ and thus $\dot{m} \propto \dot{\gamma}^{1 / 2}$.

The analysis of the diffusion mass flux has to be understood as an elementary discussion of the problem rather than an in-depth examination of all aspects. By assuming a flat interface we neglect the influence of surface tension on the concentration at the liquid-gas interface (smaller mass flux) and the increase of the surface area of the nucleus during its growth (larger mass flux). These effects counteract each other. We also neglect the interaction of the growing surface nucleus with the concentration boundary layer that probably leads to a smaller concentration 
Case I

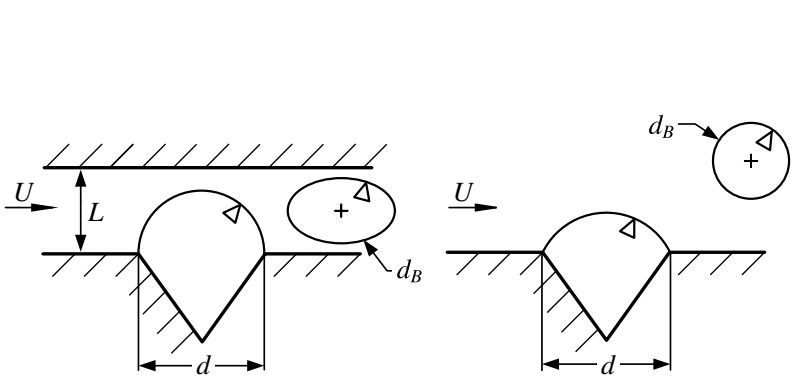

Case III

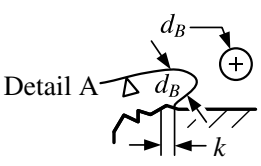

FIGURE 5. Sketch of three types of bubble detachment.

boundary layer thickness and thus to higher mass fluxes. In total we expect the model equation (2.14) to be a lower bound for the actually occurring diffusion mass flux. A quantification of the mentioned effects is hardly possible on the basis of our approach. A numerical solution of the transient advection-diffusion equation coupled with calculations of the bubble growth and the velocity profile could deliver more detailed results in the future. Nevertheless, our approach captures the relevant physical content of the problem, as the comparison with experimental results in $\S 4$ demonstrates.

\subsection{Bubble detachment}

A surface nucleus sitting in the wall of a container in a quiescent liquid, e.g. drinking glass or at a particle in the liquid bulk, grows until it reaches a critical size and a bubble detaches. The buoyancy force overcomes the capillary force. This case has been investigated extensively, cf. Fritz (1935), Jones et al. (1999), Liger-Belair (2005). In the present paper we consider the detachment of bubbles from micro crevices in a fluid flow and investigate the influence of the related forces.

We investigate three different cases and end up with three relations for the dimensionless bubble diameter of the form

$$
\delta:=\frac{d_{B}}{d}, \quad \delta=\delta(W e),
$$

with Weber number

$$
W e:=\frac{\varrho \dot{\gamma}^{2} d^{3}}{S} .
$$

In contrast to the static case, the buoyancy force only plays a minor role, especially when crevices on a microscale are considered. The capillary force is balanced by a dynamic force proportional to $\varrho U^{2} b^{2}$ with a characteristic velocity $U$ and a characteristic length $b$. The dynamic force is made up of two effects: form drag and shear lift due to an unsymmetrical flow around the surface nucleus. Depending on the flow situation, the characteristic length $b$ is the characteristic length of the device $L$ (e.g. diameter of a pipe or height of a gap), the diameter of the crevice $d$, the bubble diameter $d_{B}$ or a typical length of a surface roughness element $k$ describing the microscopic geometry of the edge of the crevice, see figure 5. The detaching bubbles are characterised by their diameter $d_{B}$. In the case of spherical bubbles $d_{B}$ is the bubble diameter. In the case of non-spherical bubbles $d_{B}$ is the equivalent diameter of a sphere with the same volume, $d_{B}=\left(6 V_{B} / \pi\right)^{1 / 3}$ with bubble volume $V_{B}$. We further assume that the surface nucleus has a mobile surface, resulting in 
negligible friction at the liquid-gas interface. Thus, the viscous force acting on the surface nucleus is considered to be small. The concept of mobile surfaces has been studied extensively in the context of rising gas bubbles (Lochiel \& Calderbank 1964; Clift, Grace \& Weber 1978; Duineveld 1995; Peters \& Els 2012). If the liquid-gas interface is immobilised, e.g. by surfactants or organic material, the viscous force has to be taken into account.

Our analysis of bubble detachment from surface nuclei is based on two competing forces, the dynamic force and the capillary force. It should be noted that there are other major research areas in which the detachment of bubbles is of importance: (i) bubble detachment from wall orifices in liquid cross-flows due to gas injection (e.g. Clift et al. 1978; Nahra \& Kamonati 2003; Duhar \& Colin 2006) and (ii) bubble detachment in flow boiling (e.g. Chen, Pan \& Ren 2012). The main difference to our approach is the need to consider additional forces in the above-mentioned cases, see cited works. In the case of bubble detachment from wall orifices an additional force proportional to the squared gas injection flow rate, called the momentum flux force, has to be considered. In the case of flow boiling a growth force of the surface nucleus as well as an added-mass force have to be taken into account. Both forces come into play because of the rapid expansion of the surface nucleus due to the evaporation of the liquid. Especially the growth force can be dominant, cf. Chen et al. (2012). In our case the growth rate of the surface nucleus is relatively small and can be neglected.

To complete our picture of the diffusion-driven nucleation we consider the three cases sketched in figure 5.

Case I: The size of the detaching bubble is of the same order of magnitude as the surface nucleus and the characteristic length of the device, $d_{B} \sim d \sim L$. Thus $d_{B}$ depends on both the size of the surface nucleus and the characteristic length of the device. This case has been investigated by Peters \& Honza (2014) and Groß, Ludwig \& Pelz (2015) in a laminar radial gap flow. In laminar flows the typical velocity is proportional to the product of wall shear rate and gap height, $U \propto \dot{\gamma} L$. The cross-section area, where the dynamic force is acting, is $b^{2} \propto d L$. The volume of the detaching bubble is $d_{B}^{3} \propto d^{2} L$. Thus the dynamic force is proportional to $\varrho \dot{\gamma}^{2} d^{3} d_{B}^{9} / d^{8}$. With the capillary force being proportional to $S d$ one obtains the specific form of equation (2.15)

$$
\delta^{3}=\left(\epsilon_{I} W e\right)^{-1 / 3},
$$

where $\epsilon_{I}$ is a dimensionless constant.

Case II: The size of detaching bubble is of the same order of magnitude as the size of the crevice, and both are significantly smaller than the characteristic length of the device, $d_{B} \sim d \ll L$. Thus the size of the bubble only depends on the size of the crevice. With the typical velocity $U \propto \dot{\gamma} d_{B}$, the cross-section area $b^{2} \propto d_{B}^{2}$, and the capillary force being proportional to $S d$, one gets

$$
\delta^{3}=\left(\epsilon_{I I} W e\right)^{-3 / 4},
$$

with the dimensionless constant $\epsilon_{I I}$. As we will show later, this case describes the most frequently observed mechanism in our experiments with water.

Case III: The detaching bubble is smaller than the surface nucleus and the characteristic length of the device, $d_{B} \ll d$ and $d_{B} \ll L$. In this case the bubble diameter $d_{B}$ cannot be a function of these lengths. The bubble size depends on the microscopic characteristics of the crevice represented by length $k$, see figure 5 . We observed this detachment mechanism only at crevices with relatively large diameters. For example, this detachment mechanism has been observed in a previous study 
with silicone oil (Groß et al. 2016) as well as in the streak cavitation experiments conducted in Lausanne, cf. figure 2 and Guennoun et al. (2003), Guennoun (2006). The typical velocity and the cross-section area are the same as in case II. However, the capillary force does not act over the hole crevice but rather on a length proportional to $d_{B}$. Hence we find

$$
\delta^{3}=\left(\epsilon_{I I I} W e\right)^{-1},
$$

with the dimensionless constant $\epsilon_{I I I}$.

For cavitating flows in pumps, turbines, nozzles or on propellers, cases II and III are of particular importance, whereas case I could be of importance for cavitation in journal bearings, narrow gaps of seals, as well as in microfluidic devices or biofluidic situations.

To complete the analysis, the bubble mass is required. For an ideal gas with the constitutive equation $p_{B}=\varrho_{B} R T_{B}$ one obtains

$$
m_{B}=\frac{\pi}{6} \frac{p_{B} d_{B}^{3}}{R T_{B}}=\frac{\pi}{6} \frac{p_{B} d^{3}}{R T} \delta^{3},
$$

with the temperature of the bubble being equal to the temperature of the surrounding liquid, $T_{B}=T$. The initial relation $f=\dot{m} / m_{B}$ together with (2.13), Henry's law $c=p \mathcal{H}$ and the ideal gas constant $\mathcal{R}=R M$ yields the Strouhal number relation

$$
S r:=\frac{f d^{2}}{\mathcal{D}} \approx 1.26 \Lambda \frac{c_{\infty} / \mathcal{H}-p_{N}}{p_{B}} P e^{1 / 3} \delta^{-3},
$$

with

$$
\Lambda:=\mathcal{R} T \mathcal{H}
$$

being the dimensionless solubility of gas in the liquid. For $p_{B} \approx p_{N}$ this reduces to

$$
S r \approx 1.26 \Lambda \zeta P e^{1 / 3} \delta^{-3},
$$

with

$$
\delta^{3}(W e)= \begin{cases}\left(\epsilon_{I} W e\right)^{-1 / 3} & \text { for case I, } \\ \left(\epsilon_{I I} W e\right)^{-3 / 4} & \text { for case II, and } \\ \left(\epsilon_{I I} W e\right)^{-1} & \text { for case III. }\end{cases}
$$

The absolute values of the dimensionless constants $\epsilon_{I}, \epsilon_{I I}$ and $\epsilon_{I I I}$, and the limits of validity are not known. One has to keep in mind that our analysis is based on simple concepts for the capillary force and the dynamic force. We do not consider contact line dynamics, the influence of contact angles and the role of the edge of the crevices for the detachment process. The length over which the capillary force is acting might differ from the length we assume. For a case II detachment the capillary force is calculated with the circumference of the crevice, which refers to an ideal situation, i.e. a symmetrical surface nucleus adhered to the edge of the crevice. To completely solve these issues, more experimental and numerical investigations on contact line dynamics, the role of the contact angles and the micro-structure of the crevices are needed, especially for large shear rates. In other research fields, e.g. heterogeneous nucleation in acoustic cavitation (Atchley \& Prosperetti 1989; Borkent et al. 2009), the growth of surface nano-bubbles (Lohse \& Zhang 2015) and detachment of vapour 
bubbles of smooth surfaces in flow boiling (Klausner et al. 1993; Chen et al. 2012), the role of contact line dynamics and contact angle are understood far more and ought to be applied to the situation discussed here. In addition we do not consider the effect of the shape of the surface nucleus on the drag and lift coefficients, which are hidden in the dimensionless constants as well. Taking all these issues into account, we cannot yet specify the values of the dimensionless constant in advance. In the following we validate the asymptotic relations of (2.23) experimentally.

\subsection{Summary of key findings so far}

The nucleation rate can be described by developing two models for the diffusion mass flux and the mass of the detaching bubbles. The diffusion mass flux is derived using boundary layer theory. The bubble mass is derived using a simplified balance of forces. The following list summarises the most important findings of our analysis. (i) There is a linear dependency of the nucleation rate on the supersaturation of the liquid. (ii) The nucleation rate depends on the Péclet number being relevant for the mass flux as well as on the Weber number being relevant for the mass of the detaching bubbles. We provide three asymptotic expressions that allow the calculation of the Strouhal number depending on the detachment mechanism. With equation (2.23) one can determine how the nucleation rate depends on the shear rate for the three detachment mechanisms. One obtains the relations $f \propto \dot{\gamma}, f \propto \dot{\gamma}^{11 / 6}$ and $f \propto \dot{\gamma}^{7 / 3}$, respectively, for the three cases. Using the dimensionless mass flux relation $S h \propto P e^{\prime 1 / 2}$ for a bulk flow allows the calculation of upper bounds for the nucleation rate. With the results of $\S \S 2.1$ and 2.2, i.e. $\dot{m} \propto \dot{\gamma}^{1 / 3}$ and $m_{B} \propto \dot{\gamma}^{-2 / 3}, m_{B} \propto \dot{\gamma}^{-3 / 2}$ or $m_{B} \propto \dot{\gamma}^{-2}$, it is obvious that the influence of the shear rate on the mass of the bubbles is much greater than on the diffusion mass flux into the surface nucleus. (iii) The nucleation rate is proportional to $\Lambda:=\mathcal{R} T \mathcal{H}$, which is a dimensionless quantity describing the capability of the liquid to dissolve gas. It can be interpreted as the ratio between the volume occupied by a given mass of gas at a given pressure and the volume of liquid that could be saturated with that given mass. This factor is varied only marginally in cavitation experiments, e.g. due to temperature variations, and can be set to a constant value if the experiments are conducted with the same liquid. Of course, $\Lambda$ may vary over several orders of magnitude when switching from water to oil or when different gases are investigated, e.g. carbon dioxide in water. (iv) The mass of the non-condensable gas inside of a detaching spherical bubble is proportional to the pressure $p_{B}=p_{\infty}+4 S / d_{B}-p_{v}$ with surrounding pressure $p_{\infty}$, vapour pressure $p_{v}$ and surface tension $S$. In the case of small bubbles or low surrounding pressures the influence of surface tension and vapour pressure cannot be neglected. The effect of surface tension increases the bubble mass, resulting in a decrease of the nucleation rate. In the case of low surrounding pressures the vapour pressure has to be taken into account, leading to a decrease of the bubble mass and thus to an increase of the nucleation rate. (v) The effects of hydrophobic and hydrophilic surfaces are not considered so far. Contact line dynamics and different contact angles may influence the detachment mechanism and can be taken into account by a variation of the dimensionless constants $\epsilon_{I}, \epsilon_{I I}$ and $\epsilon_{I I I}$. In the end, we expect that the microscopic geometry of the edge of the crevices plays a dominant role and surface energy is only of secondary importance.

\section{Experimental set-up}

Experiments on cavitation are often carried out in complex test facilities since the results are to be applied on likewise complex machines such as pumps, propellers or 


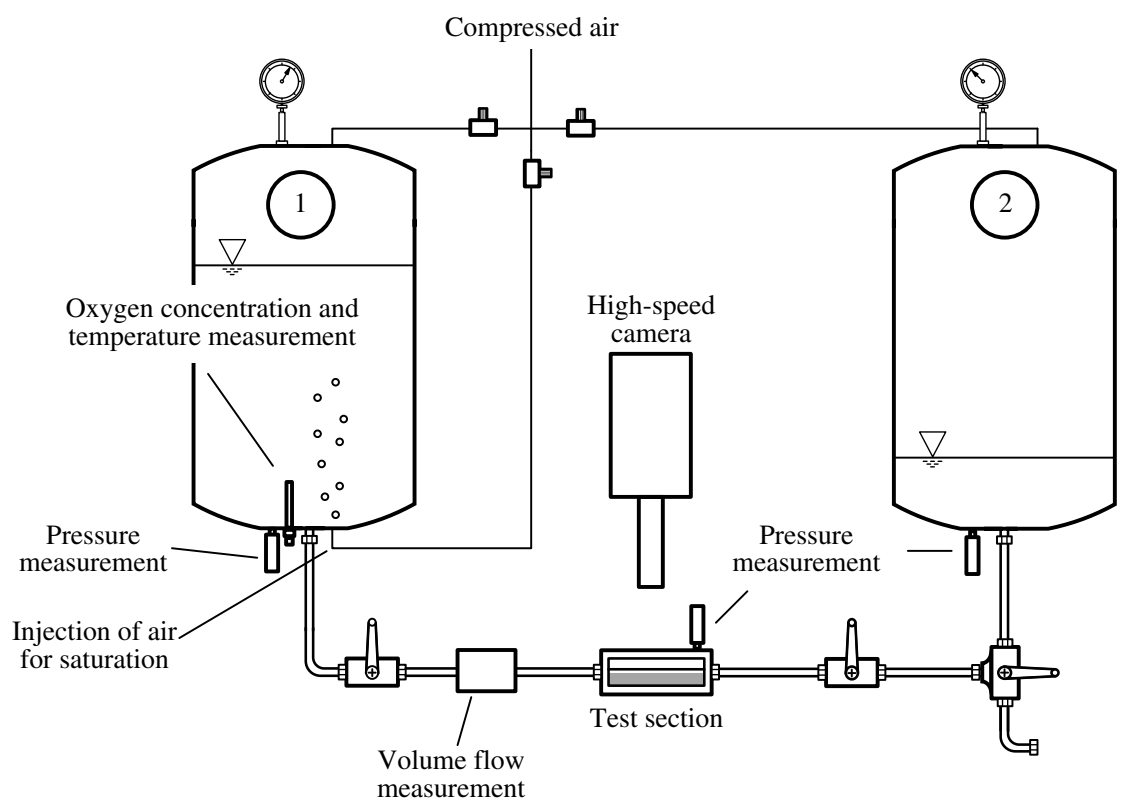

FIGURE 6. Sketch of experimental set-up.

valves. Due to the complexity and size of these test facilities, i.e. cavitation tunnels, there often is a limited accessibility to the working section, resulting in extensive experimental procedures and time-consuming conversion work. Usually the focus is on large-scale cavitation phenomena such as sheet and cloud cavitation, cf. Pelz et al. $(2014,2017)$. Without a doubt, small-scale phenomena such as nucleation are difficult to study in these experimental set-ups. The experimental set-up we use for our nucleation experiments is kept simple so that a good accessibility (mechanical and optical) is ensured and we can easily control the experimental parameters. In technical applications a bubble might show an exponential growth (cavitation) right after its detachment from a surface nucleus. The almost simultaneous occurrence of diffusion-driven nucleation and cavitation, cf. figure 2, complicates both the execution of the experiments and the analysis of the collected data. For this reason it is advantageous to separate these two phenomena. In fact, in our experiments the pressure is way above vapour pressure so that cavitation cannot be observed. The formation of bubbles can only be caused by diffusion.

The focus of the experiment is on the measurement of the nucleation rates, the growth of the surface nuclei and the detachment process by means of high-speed visualisation. Figure 6 shows a sketch of the experimental set-up, consisting of two tanks with a capacity of 501 each, the test section, piping, gas supply, various measuring instruments, the high-speed camera system and a submicron filtering unit to filter out small particles (not shown in the sketch). The fluid flow is driven by a pressure difference between the two tanks that can be adjusted using compressed air. The liquid, usually tap water, flows from tank 1 through the piping and the test section, where the surface nuclei are located, into tank 2 . We measure the pressure in both tanks, the pressure in the test section, the oxygen content and the temperature of the liquid in tank 1. The flow rate is measured with a magneto-inductive flow meter. High-speed visualisations are conducted using an IDT Motion Pro Y7 S3 camera 


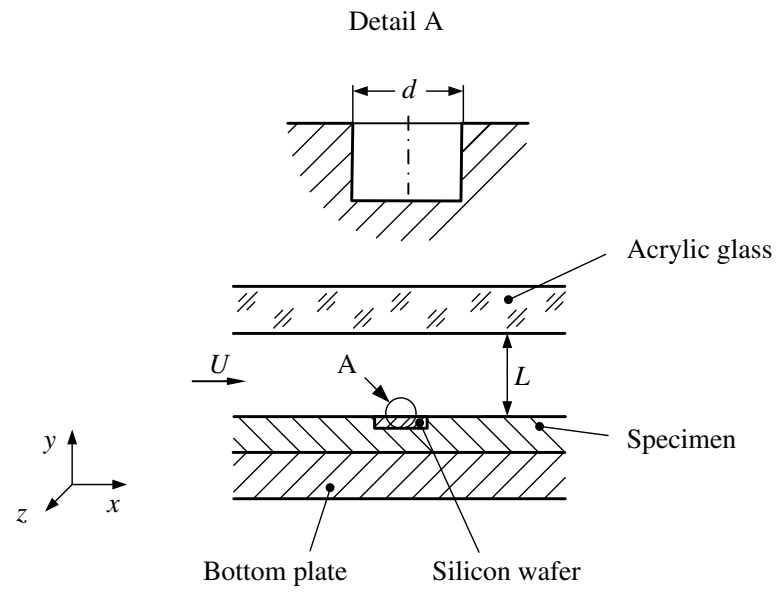

FIGURE 7. Sketch of pipe with rectangular cross-section. Blind holes etched in silicon wafer work as surface nuclei. The high-speed camera is mounted either in the top ( $x-z-$ plane) or the side view ( $x-y$-plane) perspective.

with a resolution of $1920 \times 1080$ pixels and a maximum frame rate of 10600 f.p.s and stroboscopic lighting. It is essential to use a long-distance microscope to visualise the micrometre-sized crevices and the detaching bubbles. To eliminate motion blur of the moving bubbles it is necessary to use exposure times of microseconds, which is in conflict with sufficient lighting. Thus, the measurement of the nucleation rate is much easier and more precise than the measurement of the diameters of the moving bubbles. In order to make a statement about the bubble size possible, the size of the surface nuclei is analysed immediately prior to the detachment.

The test section we use is a pipe with a rectangular cross-section, see figure 7 . The rectangular pipe has a width of $w=30 \mathrm{~mm}$ and an adjustable gap height $L$. Distance plates are inserted into the test section to change the gap height between 1.8 and $3.8 \mathrm{~mm}$ in discrete steps. We achieve flow rates of up to $101 \mathrm{~min}^{-1}$. By closing a control valve the flow rate can be set to zero. We expect the velocity profile to be laminar. Thus, the shear rate at the wall is $\dot{\gamma}=6 Q / L^{2} w$, with flow rate $Q$. We achieve shear rates of the order of magnitude of $10^{2} \mathrm{~s}^{-1}-10^{3} \mathrm{~s}^{-1}$ and supersaturations of the order of magnitude of 0.1-1. In comparison to technical relevant flows in pumps, turbines, propellers or valves, the shear rates are still relatively small.

Since the lid and the sidewalls are made of acrylic glass, high-speed visualisations from the top and side view perspective are possible. The observed crevices are cylindrical blind holes with diameters of $25 \mu \mathrm{m}, 50 \mu \mathrm{m}$ and $100 \mu \mathrm{m}$ etched into square-shaped silicon wafers with a edge length of $10 \mathrm{~mm}$. The depth of the blind holes is approximately $20 \mu \mathrm{m}$, while the silicon wafer has a thickness of $0.5 \mathrm{~mm}$. The silicon wafers were produced and kindly given to us by Dr David Fernandez Rivas from the Mesoscale Chemical Systems group at University of Twente, Netherlands. See Fernandez Rivas et al. (2010) and Zijlstra et al. (2015) for further information about the production. The ratio of gap height of the test section to diameter of the surface nuclei is at least 18 . The maximum ratio is 152 . Thus, we expect the nucleation process not to be influenced by the height of the gap. The silicon wafers are mounted on a specimen that is inserted into the test section.

Before starting a series of measurements one has to ensure that the silicon wafers are very clean and dust particles have been removed. The crevices are filled with gas 
by simply filling the test section with liquid, cf. Bankoff (1958). Small amounts of gas are entrapped in the crevices and work as surface nuclei. The saturation of the liquid in tank 1 is adjusted by injecting compressed air at the bottom of the tank. The measurement of the oxygen content is used to ensure that the liquid is saturated at a specific pressure. One of the most important qualities of the set-up is that the supersaturation in the test section can be varied while the other parameters can be kept constant. The pressure in the test section is used to determine the pressure of noncondensable gas in the surface nuclei $\left(p_{N}\right)$, while the pressure in tank 1 corresponds to the saturation pressure of the liquid $\left(p_{\infty}\right)$, which is controlled with an oxygen sensor. The supersaturation of the liquid in the test section can be determined with $\zeta:=p_{\infty} / p_{N}-1$, cf. equation (2.3). Increasing the pressure level of the set-up and keeping the pressure difference between the tanks constant, leads to a constant flow rate (for a given gap height) while the supersaturation changes. On the other hand it is possible to keep the supersaturation constant while the flow rate changes. If the pressure level is changed rapidly, the liquid in tank 1 is still saturated at the saturation pressure that has been adjusted before. Keeping the pressure in the test section constant results in a constant supersaturation and a variable flow rate that depends on the pressure difference between the tanks.

\section{Experimental results and discussion}

To get an extended understanding of the nucleation process we investigate the nucleation rates and the volume of detaching bubbles by means of high-speed visualisation. It will be seen that the model for a case II bubble detachment, equation (2.18), and the Strouhal number relation, equation (2.23), are well validated by the experimental findings.

By way of example figure 8 shows a sequence of the growth of a surface nucleus in water and the detachment of a bubble at a micro crevice with a diameter of $d=$ $25 \mu \mathrm{m}$ in a fluid flow with a shear rate of $2026 \mathrm{~s}^{-1}$. During its growth the surface nuclei forms a spherical cap that bends over the edge of the crevice with increasing size. Prior to its detachment the surface nucleus is obviously larger than the crevice. After the detachment (not visible in the pictures) the process repeats. For the shown experiment the nucleation rate is $0.22 \mathrm{~Hz}$. The figure is a typical recording that is used for the evaluation and analysis of nucleation rates and bubble detachment.

Using the recordings from the side view perspective, it is possible to determine the volume of the surface nucleus immediately prior to the bubble detachment. Assuming that the volume of the detached bubble equals the part of the surface nucleus that protrudes into the water prior to the detachment, cf. figure 8 , frame 9 , allows the determination of the dimensionless bubble volume $\delta^{3}$. The measurement of the bubble size of course may suffer from measurement uncertainties. The bubble volume is determined using a two-dimensional projection of the surface nucleus. Deformations in the third dimension cannot be considered. Prior to the detachment the surface nucleus is in motion and shows deformations of the shape, which makes the determination of the bubble volume difficult in some cases, especially in the case of large shear rates. Furthermore, the resolution of the images complicates an exact determination of the bubble volume. Based on our evaluation, measuring uncertainties of $10 \%-15 \%$ of the bubble diameter $\delta$ are reasonable values. In addition, large fluctuations between the individual measurements have to be considered. So far, it is not known which parameters are responsible for the scattering of the data.

The grey symbols in figure 9 represent the measured dimensionless bubble volume $\delta^{3}$ plotted against Weber number $W e:=\varrho \dot{\gamma}^{2} d^{3} / S$ for different crevice diameters. The 


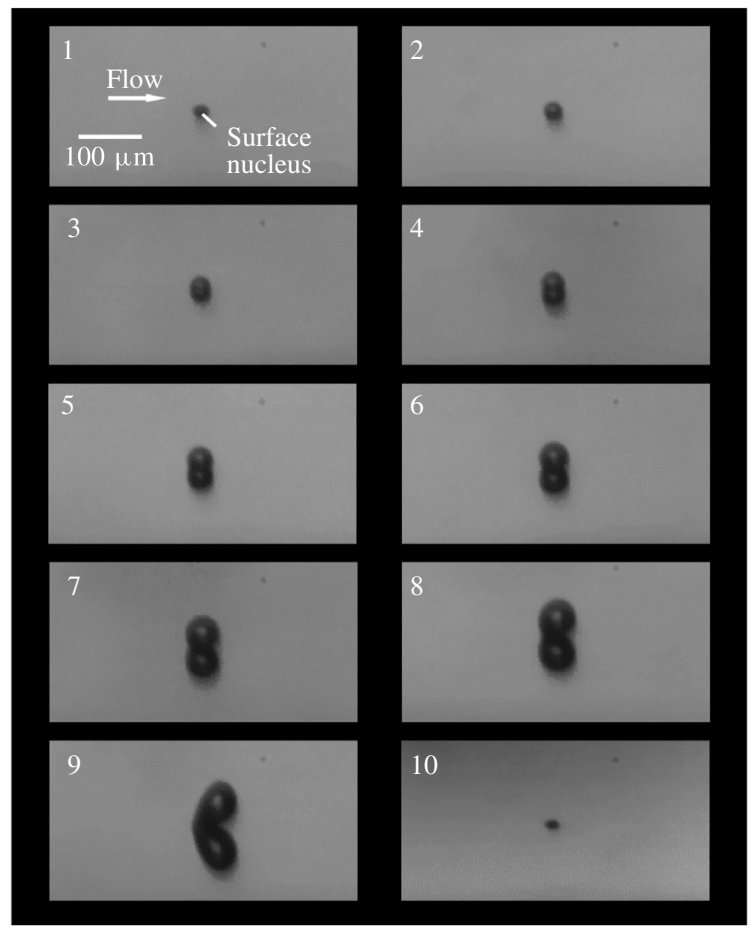

FIGURE 8. Growth of a surface nucleus and detachment of a bubble at a crevice with $d=25 \mu \mathrm{m}$ in water from the side view perspective. The flow is from left to right. The time interval between the images is $500 \mathrm{~ms}$. The surface nucleus mirrors in the silicon wafer.

different symbols mark different crevice diameters that have been evaluated. As one expects, the dimensionless bubble volume decreases with increasing Weber number, and thus increasing shear rate. In the measurements we observed case II detachments. The measured bubble volumes are in the range of $\delta^{3}=1-1 \times 10^{3}$, i.e. a range of $\delta=1-10$ for the bubble diameter.

In addition to size measurements, we also measured the nucleation rate $f$. Our experiments showed that there is a nonlinear dependence of the nucleation rate on the shear $\dot{\gamma}$ and a linear dependence on the supersaturation $\zeta$. We observed that the nucleation rate is very constant over time. We recorded at least 15 bubble detachments at each operation point so that we can calculate a reasonable standard error. Our measurements show that the $95 \%$ confidence interval of the nucleation rate is smaller than $10 \%$ of the measured value of the nucleation rate, usually better.

In figure 9 an additional and independent representation of the dimensionless bubble volume $\delta^{3}$ is presented. The black symbols in figure 9 are deduced from measurements of the nucleation rate $f$ (or Strouhal number $S r$ ) which can be measured with smaller measuring uncertainty than the bubble size. We rearrange equation (2.23), calculate

$$
\delta^{3}=\frac{1.26 \zeta \Lambda P e^{1 / 3}}{S r},
$$

and plot it against Weber number $W e=\varrho \dot{\gamma}^{2} d^{3} / S$. As one expects, the calculated dimensionless bubble volume also decreases with increasing Weber number. Together, 


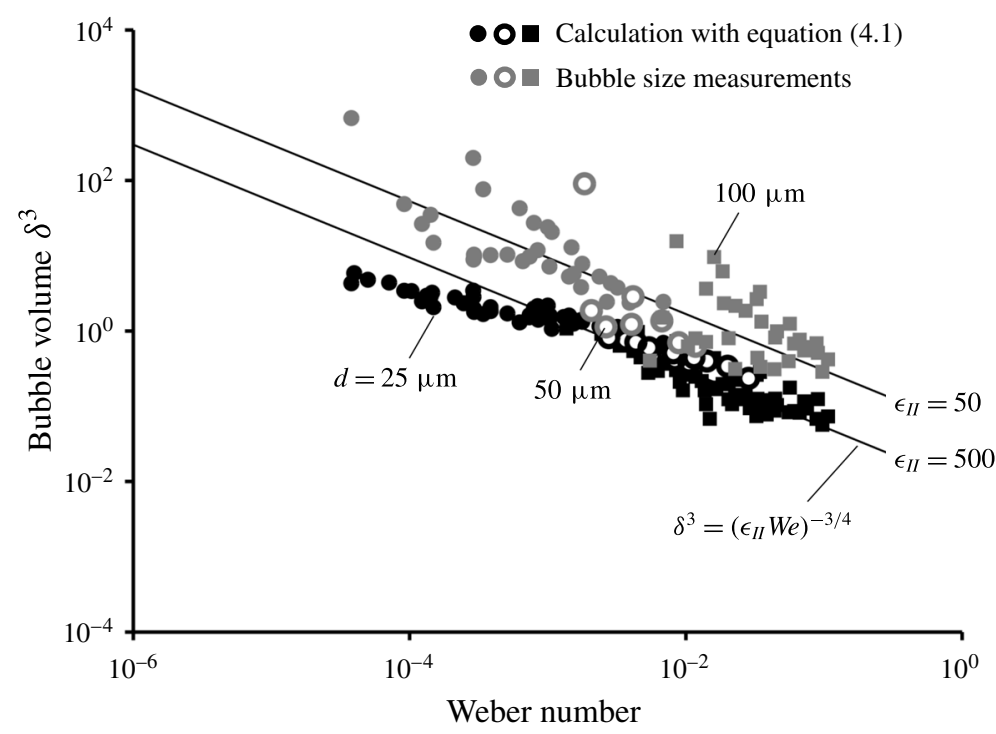

FiguRE 9. Dimensionless bubble volume $\delta^{3}$ versus Weber number We $:=\varrho \dot{\gamma}^{2} d^{3} / S$. The solid lines are calculated with equation (2.18). Grey symbols are based on measurements of the size of the detaching bubbles. Black symbols are calculated with equation (4.1) based on nucleation rate measurements. Different symbols mark different crevice diameters: $25 \mu \mathrm{m}$ filled circle, $50 \mu \mathrm{m}$ open circle, $100 \mu \mathrm{m}$ square. The ratio of crevice diameter to gap height is always smaller than $1 / 18$. The liquid is water with the properties $\varrho=998 \mathrm{~kg} \mathrm{~m}^{-3}, \mathcal{D}=2 \times 10^{-9} \mathrm{~m}^{2} \mathrm{~s}^{-1}, S=70 \times 10^{-3} \mathrm{~kg} \mathrm{~s}^{-2}$ and $\mathcal{H}=$ $7.4 \times 10^{-6} \mathrm{~mol}\left(\mathrm{~m}^{3} \mathrm{~Pa}\right)^{-1}$.

both data sets, i.e. bubble size and nucleation rate measurements, underline that our model is a reasonable approach describing diffusion-driven nucleation from surface nuclei.

The graph shows that the data of the calculated dimensionless bubble volume approach the relation $\delta^{3}=\left(\epsilon_{I I} W e\right)^{-3 / 4}$ with $\epsilon_{I I}=5 \times 10^{2}$. Based on the bubble size measurements, $\epsilon_{I I}=5 \times 10^{1}$ is a more reliable value. The main reason for the discrepancies between the two data sets are (i) large (systematic) measurement uncertainties of the bubble size measurements, which cannot be determined further so far (assumption that bubble size equals size of protruding part of surface nucleus, size measurement based on two-dimensional projection, moving surface nucleus) and (ii) uncertainties in the mass flux model. As discussed in $\$ 2.1$, the calculated diffusion mass flux can be interpreted as a lower bound. The surface area of the surface nuclei is obviously larger than the surface area of the crevices, cf. figure 8. Furthermore, the influence of the protruding surface nucleus on the thickness of the concentration boundary layer is neglected. An underestimation of the diffusion mass flux leads to smaller values of the calculated dimensionless bubble diameter, and thus to a higher value of $\epsilon_{I I}$, cf. equation (4.1). Both mentioned aspects could easily sum up to an order of magnitude in the mass flux, and thus in the calculated dimensionless bubble volume. It should be noted that for a constant Weber number a factor of ten in the dimensionless constants leads to a reduction of the bubble volume by a factor of 0.17 and to a reduction of the bubble diameter by a factor of 0.56 .

For a symmetrical surface nucleus that is adhered to the edge of the crevice one would expect $\epsilon_{I I}$ to be of the order of unity, since it represents the detachment 
criterion, which says that the dynamic force has to overcome the capillary force, which is calculated with the circumference of the crevice. This refers to an ideal situation. As mentioned in $\$ 2.2$, our detachment model does not contain all aspects of the detachment process, i.e. contact line dynamics, surface energy issues, the role of the edge of the crevice for bubble detachment, as well as the shape-dependent drag and lift coefficients. The detachment model has to be understood as a rough approach. High-speed visualisations of the growth of surface nuclei show that the surface nucleus bends over the edge of the crevice and is far from being symmetrical, cf. figure 8. The deformation of the surface nucleus depends on both its size and the shear rate at the wall. Large values of $\epsilon_{I I}$ as shown in figure 9 indicate an overestimation of the capillary force. The length over which the capillary force is acting might differ from the length we assume, i.e. the diameter of the crevice. If this length can be determined somehow, $\epsilon_{I I}$ can be scaled with $\epsilon_{I I}^{*}=\epsilon_{I I} d^{*} / d$, with $d^{*}$ being the relevant length.

In our experiments we observe nucleation rates of the order of magnitude of $0.1 \mathrm{~Hz}-1 \mathrm{~Hz}$ in the investigated range of shear rates. At first glance, these frequencies seem to be very low. One has to keep in mind that shear rates in cavitating flows can be substantially higher, i.e. $10^{4} \mathrm{~s}^{-1}-10^{5} \mathrm{~s}^{-1}$, cf. $\S 5$. On the other hand, crevices in technical applications can be at least one order of magnitude smaller than in our experiments, depending on the production process (turning, milling, grinding, casting). Diameters of $d \approx 1 \mu \mathrm{m}$ are reasonable in technical applications. If the model assumptions hold true also for higher shear rates, the results can be used to make statements on the nucleation rate also for these cases. On the basis of figure 9 one can evaluate a Strouhal number of $S r=0.1$ for a Weber number of approximately $W e \approx 10^{-3}$. In the case of a moderate supersaturation of $\zeta=1$ and $\mathcal{D}=2 \times 10^{-9} \mathrm{~m}^{2} \mathrm{~s}^{-1}$, this is related to a nucleation rate of $f=200 \mathrm{~Hz}$ for a crevice diameter of $d=1 \mu \mathrm{m}$. The required shear rate for this to happen is $\dot{\gamma}=2.6 \times 10^{5} \mathrm{~s}^{-1}$.

This example shows that the results presented can indeed be applied to technical flow problems. Of course, the transfer of the model to higher shear rates might be crucial since it has been validated only for smaller shear rates. So far, we see now restrictions that the model holds true for these cases. This has to be discussed in future investigations. In $\S 5$ the model is applied to two cases of practical interest.

\subsection{Bubble detachment as a Plateau-Rayleigh instability}

In Groß et al. (2016) we presented experimental results gained in a similar experimental set-up using the same test section. In these experiments we used silicone oil with a kinematic viscosity of $20 \times 10^{-6} \mathrm{~m}^{2} \mathrm{~s}^{-1}$ instead of water. The surface tension of the used silicone oil is $21 \times 10^{-3} \mathrm{~kg} \mathrm{~s}^{-2}$ and the density is $945 \mathrm{~kg} \mathrm{~m}^{-3}$. Relatively large blind holes with a diameter of $0.8 \mathrm{~mm}$ worked as nucleation sites. The ratios of gap height to the diameter of the surface nucleus are $L / d=1.25$ and 3.75 , respectively. Due to these small ratios, the fluid flow and thus the bubble detachment mechanism are influenced strongly. Nevertheless, we observed a detachment mechanism that might be of particular importance for some applications.

Figure 10 shows twelve high-speed images of the detachment of a bubble from a surface nucleus in the side view perspective, cf. Groß et al. (2016). The images illustrate that the detachment process is a consequence of a Plateau-Rayleigh instability (Rayleigh 1879). The detachment works as follows. The initial situation is a surface nucleus that expands over the edge of the bore and forms a cylindrical body. Small perturbations with different wavelengths that occur due to the fluid flow deform 


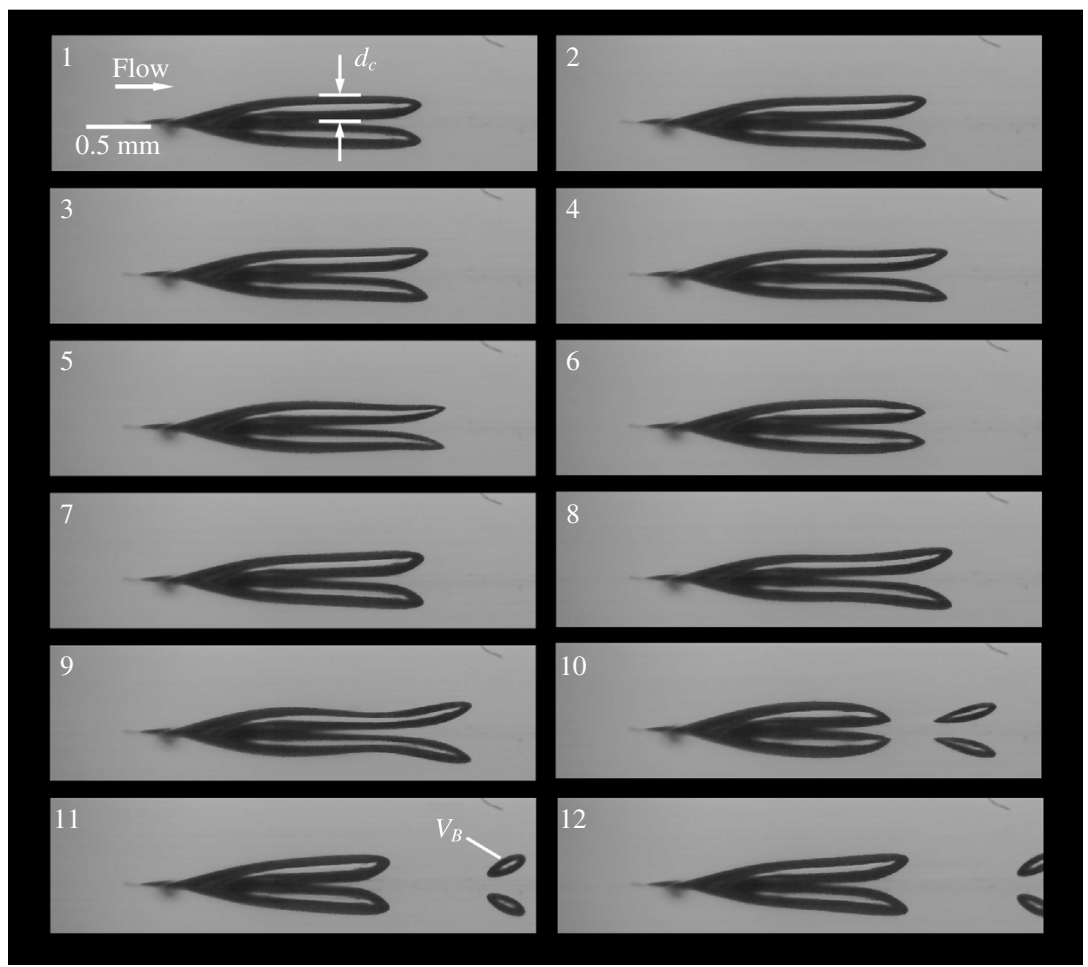

FIGURE 10. Detachment of a bubble with volume $V_{B}$ from a surface nucleus as a consequence of a Plateau-Rayleigh instability from the side view perspective. The time interval between the images is $2 \mathrm{~ms}$. The flow is from left to right. The liquid is silicone oil with a kinematic viscosity of $20 \times 10^{-6} \mathrm{~m}^{2} \mathrm{~s}^{-1}$ and a capillary constant of $21 \times 10^{-3} \mathrm{~kg} \mathrm{~s}^{-2}$. The surface nucleus and the detached bubble mirrors in the polished surface of the specimen.

the cylindrical part of the nucleus. Deformations with wavelengths smaller than a critical wavelength increase the surface of the nucleus. Wavelengths larger than the critical wavelength lead to a reduction of the surface. Regarding to Rayleigh's work principle, the nucleus is stable to deformations that increase the surface since work would have to be done. Deformations that decrease the surface will not be damped but increased because the system attempts to reach an energetically favourable state. For a cylindrical body with diameter $d_{c}$ with an infinite length the critical wavelength is its circumference $\pi d_{c}$ (Rayleigh 1879).

The largest wavelength that occurs approximately equals the length of the cylindrical part of the nucleus. The length of the nucleus, and thus the largest wavelength, increases while the surface nucleus is growing. The nucleus starts to oscillate up and down as soon as the critical length is reached. Since the perturbations are not attenuated, the cylindrical part of the surface nucleus deforms so strongly that it constricts and a bubble detaches. The Plateau-Rayleigh instability is a geometrical criterion. Of course, the bubble shape and the expansion over the edge of the bore are results of the acting forces.

Figure 11 shows how the volume of the detached bubbles depends on the shear rate (graph $a$ ) as well as on the cylindrical part of the surface nucleus (graph $c$ ). As 

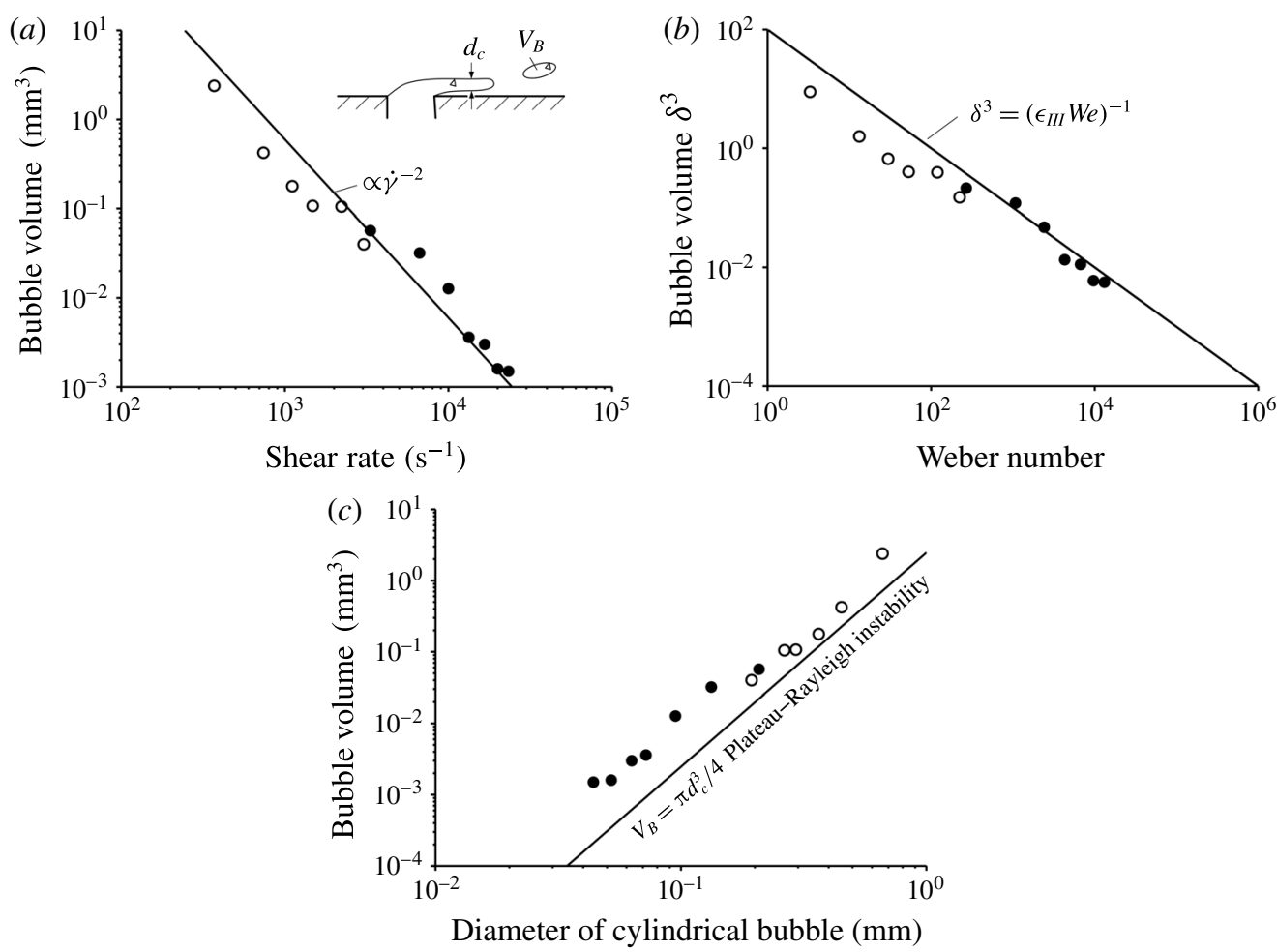

FIGURE 11. (a) Volume of detached bubble versus shear rate. (b) Dimensionless bubble volume $\delta^{3}$ versus Weber number. The solid line is calculated with equation (2.19) and $\epsilon_{I I I}=0.01$. (c) Volume of detached bubble versus diameter of the cylindrical part of the surface nucleus. The solid line marks the critical volume $V_{B}=\pi^{2} d_{c}^{3} / 4$. The open circles mark measurements with a gap height of $3 \mathrm{~mm}$. The filled circles mark measurements with a gap height of $1 \mathrm{~mm}$. The liquid is silicone oil with the properties $\varrho=945 \mathrm{~kg} \mathrm{~m}^{-3}$ and $S=21 \times 10^{-3} \mathrm{~kg} \mathrm{~s}^{-2}$.

one expects, the volume of the detached bubbles decreases with increasing shear rate. The graphs illustrate that the detachment process depends only marginally on the gap height. Figure 11(a) also confirms the validity $S r \propto W e P e^{1 / 3}$, equation (2.23) case III. The model implies $m_{B} \propto V_{B} \propto \dot{\gamma}^{-2}$, which can also be found in the graph. At first glance this finding is somehow surprising, since the detachment mechanism described here is not driven by forces but rather a stability limit based on surface energy. In fact, the growth of the nucleus and the formation of the shape follow the same physical law as the detachment mechanism described in $\S 2.2$. The only difference is the trigger that causes the detachment (balance of forces or Rayleigh-Plateau instability). To underline this finding, figure $11(b)$ shows the dimensionless bubble volume $\delta^{3}$ plotted against Weber number. The solid line is calculated with equation (2.19) and the dimensionless constant is determined as $\epsilon_{I I I}=0.01$. One clearly sees that the experimental results are well described by the asymptote $\delta^{3}=\left(\epsilon_{I I I} W e\right)^{-1}$.

It should be noted that the value of $\epsilon_{I I I}$ is very small compared to the values of $\epsilon_{I I}$ discussed in the previous section. This is due to an overestimation of the dynamic force in the present case. The surface nucleus shows an 'streamlined' shape leading to a reduction of drag. Hence, it is not surprising that $\epsilon_{I I I}$ has a relatively small value. 
Figure 11(c) illustrates how the volume of the detached bubbles increases with increasing diameter of the cylindrical part of the nucleus. As mentioned before, the surface nucleus becomes unstable when it reaches a critical size, and perturbations with wavelengths larger than its diameter occur. The straightforward approach to calculate the volume of the detached bubbles is to calculate the volume of a cylinder whose length equals its circumference, $V_{B}=\pi^{2} d_{c}^{3} / 4$. The solid line in figure 11(c) represents this calculation. The volume of the detached bubble is proportional to the cube of the diameter of the cylindrical part. The measured values lie only slightly above the solid line.

Our experiments with water and silicone oil demonstrate that there are a variety of detachment mechanisms that might occur, cf. figures 8 and 10. At first sight, the Plateau-Rayleigh instability seems not to be of practical importance, especially for water flows. However, there is experimental evidence that this detachment mechanism also occurs in hydrodynamic cavitation. Guennoun (2006) observed the detachment of bubbles from a surface nucleus attached to a surface irregularity (see figure 4.7 of his work). The detachment behaviour looks very similar to the behaviour described here (Martijn van Rijsbergen, personal communication, February 2, 2016).

\section{Application to technical flow problems}

In the previous sections we demonstrated that nucleation rates can be calculated on the basis of the approach $f=\dot{m} / m_{B}$. We showed that the three correlations $S r \propto$ $\zeta W e^{1 / 3} P e^{1 / 3}, S r \propto \zeta W e^{3 / 4} P e^{1 / 3}$ and $S r \propto \zeta W e P e^{1 / 3}$ are useful to calculate nucleation rates occurring at micro crevices. To underline the importance of our findings we discuss our approach using examples of practical interest.

First, we want to take a look on the streak cavitation experiments conducted in Lausanne, cf. figure 2. The theoretical findings indicate that the shear rate plays a crucial role in the described nucleation process. The shear rate on a hydrofoil can be determined using the theory for the evolution of the boundary layer thickness on a flat plate, $\delta_{U}=5 x / R e_{x}^{1 / 2}$ with coordinate $x$, Reynolds number $R e_{x}=U_{\infty} x / v$ and velocity of the undisturbed flow $U_{\infty}$ (Schlichting \& Gersten 2006). Using $\dot{\gamma} \approx U_{\infty} / \delta_{U}$ we get $\dot{\gamma} \approx 1 / 5 \sqrt{U_{\infty}^{3} /(x v)}$. Guennoun et al. (2003) conducted experiments with $U_{\infty}=$ $20 \mathrm{~m} \mathrm{~s}^{-1}$ and observed nucleation at $x=1 \mathrm{~mm}$. At the nucleation site the shear rate has a value of $5.7 \times 10^{5} \mathrm{~s}^{-1}$, and is thus two orders of magnitude larger than the shear rates observed in our experiments. Guennoun observed nucleation rates of up to $5 \mathrm{kHz}$. Using $f=\dot{m} / m_{B}$ and the correlation for the mass flux equation (2.13) one obtains a bubble diameter of $d_{B} \approx 18.1 \mu \mathrm{m}$ for a moderate supersaturation of $\zeta=1$. The bubble diameter increases with $\zeta^{1 / 3}\left(\mathcal{R}=8.314 \mathrm{~J}(\mathrm{~mol} \mathrm{~K})^{-1}, T=293.15 \mathrm{~K}, \mathcal{H}=\right.$ $\left.7.4 \times 10^{-6} \mathrm{~mol}\left(\mathrm{~m}^{3} \mathrm{~Pa}\right)^{-1}\right)$. On the basis of (2.23) and assuming a case III detachment we obtain $\epsilon_{I I I}=3.67 \times 10^{-2}$ with $W e=4.6 \times 10^{6}$. This value is surprisingly close to the value of $\epsilon_{I I I}=10^{-2}$ determined in the previous section. In the light of this finding a case III detachment seems to be unlikely in the case of micro crevices, due to the large required shear rates.

A second practical application of our model is a plausibility check of EulerLagrange simulations of cavitating flows where surface nucleation is considered. Recent computational fluid dynamics (CFD) studies on sheet and cloud cavitation underline the relevance of nucleation in the context of hydrodynamic cavitation. Chahine and his group (e.g. Hsiao, Ma \& Chahine 2014; Chahine 2015; Ma, Hsiao \& Chahine 2015) investigated sheet and cloud cavitation using an Euler-Lagrange model where cavitation nuclei and their bubble dynamics are considered. The nuclei 
are created at nucleation spots on the surface of the investigated objects. The authors used a nucleation rate of $22 \mathrm{kHz}$ in their simulations. The model works without any empirical mass exchange models that are non-physical and need to be calibrated. Although this approach is computationally expensive in comparison to other cavitation models (e.g. Schnerr \& Sauer 2001; Singhal et al. 2001), it is physically more consistent for representing the processes of sheet and cloud cavitation. Since there is only a little influence of the bubble radius on the numerical results, the predefined initial bubble radius is set to be $10 \mu \mathrm{m}$. The nucleation rate and the initial nuclei radius used in the simulations are not experimentally validated. One has to keep in mind that the size of the nucleation spots are of the order of magnitude of the surface roughness or the size of surface irregularities (e.g. shrinkage cavities), and thus of orders of magnitude from 0.1 to $10 \mu \mathrm{m}$. It would be very unlikely that the diameters of the detaching bubbles are orders of magnitude larger than the size of the nucleation spots for the large shear rates and large nucleation rates occurring in cavitating flows. We can use our model to check the predefined bubble diameter on plausibility.

Assuming a nucleation spot with $d=10 \mu \mathrm{m}$ we can use equation (2.13) to calculate a shear rate of $1.2 \times 10^{18} \mathrm{~s}^{-1}$ for a moderate supersaturation of 1 . This shear rate is unphysically large. We now use equation (2.23) with $\epsilon_{I I}=5 \times 10^{2}$, cf. figure 9 , and calculate the shear rate for a case II detachment to be $\dot{\gamma} \approx 1.3 \times 10^{6} \mathrm{~s}^{-1}$, which is still large (the properties of the liquid are listed above). The Weber number then is $W e \approx 25.3$. Equation (2.18) then gives a bubble diameter of $d_{B} \approx 0.9 \mu \mathrm{m}$, which is small but a more reliable value than $20 \mu \mathrm{m}$. In summary, diffusion-driven nucleation of bubbles with a diameter of $20 \mu \mathrm{m}$ with a frequency of $22 \mathrm{kHz}$ seems implausible in the light of our findings.

In our model we only consider single surface nuclei sitting in a well-defined crevice. In technical applications there are an uncountable number of nucleation spots located on a surface with a variety of geometries, e.g. steps (Pelz et al. 2014), surface irregularities or roughness elements (van Rijsbergen \& van Terwisga 2011). In Pelz et al. (2017) we discussed the nucleation process in the context of sheet and cloud cavitation originating from an artificial roughness. Taking into consideration that free-stream nuclei 'activate' the artificial roughness and form a large liquid-gas interface, one can conclude that diffusion-driven nucleation is responsible for the production of the vast number of bubbles forming the sheet cavity. Large shear rates together with a sufficiently large liquid-gas interface are the key elements here.

Of course, the process of diffusion-driven nucleation in technical flows is a very complex phenomenon. Different nucleation sites on surfaces could interact with each other, and could combine and form a larger surface nucleus. In addition, detaching bubbles could influence each other or could cause the bubble detachment at surface nuclei further downstream. The nucleation process is influenced by an usually unknown local supersaturation and might be affected by the wettability of the surface. Furthermore, the surface tension and the vapour pressure of the liquid could play an important role as well as turbulence and vortex formation. This work can serve as a first step for further investigations on diffusion-driven nucleation in the context of hydrodynamic cavitation. In the light of the findings presented, the role of diffusion must be reassessed.

\section{Conclusion}

There is no doubt that free-stream nuclei and surface nuclei play an important role for the inception and appearance of cavitating flows. Cavitation nuclei work as 
weak spots in the liquid and allow its rupture under technically relevant pressures. The theories and models of nuclei and their stabilisation are well established and recognised in the cavitation community. Nevertheless, there is a lack of knowledge about the principles of diffusion-driven nucleation from surface nuclei in the context of hydrodynamic cavitation. In the present paper we investigate the formation of bubbles that might serve as cavitation nuclei at pre-existing gas cavities (surface nuclei) in a supersaturated liquid due to gas diffusion. The produced bubbles might act as cavitation nuclei when entering a low-pressure region.

The self-excited cyclic process of diffusion-driven nucleation is analysed and new theoretical considerations are presented and validated with experimental results. The nucleation rate is expressed as the ratio of the mass flux that diffuses into the surface nucleus and the mass of the detaching bubbles, $f=\dot{m} / m_{B}$. Therefore, it is expedient to treat these two aspects, i.e. the two phases of the cyclic process, separately. The mass transfer is modelled by solving an advection-diffusion equation while the bubble detachment is treated with a balance of forces. The first model shows that the mass transfer is intensified by the fluid flow, as one expects. The dimensionless mass transfer (Sherwood number) is proportional to the Péclet number to the power of one third and depends linearly on the supersaturation of the liquid $\zeta$. The second model shows that the size of the detaching bubbles is a function of the Weber number. Depending on the detachment mechanism one obtains three asymptotes $S r \propto \zeta \Lambda W e^{n} P e^{1 / 3}$ with $n=1 / 3,3 / 4,1$ for the nucleation rate with dimensionless gas solubility $\Lambda$. The results of the calculation are in good agreement with experimental findings gained in generic experiments. The models serve well for the analysis of streak cavitation. In addition, the models can be used to check the plausibility of nucleation rates and bubble diameters used in Euler-Lagrange simulations of cavitating flows. The transfer of the findings to other cavitation regimes such as sheet and cloud cavitation is conceivable. The discussion about diffusion-driven nucleation leads to a new assessment of the role of diffusion in hydrodynamic cavitation.

\section{Acknowledgements}

We would like to thank Professor Dr F. Peters (Ruhr-Universität Bochum), for valuable hints regarding the experimental set-up, and Dr D. Fernandez Rivas (Mesoscale Chemical Systems group, University of Twente), who supplied us with the silicon wafers.

\section{REFERENCES}

Andersen, A. \& MørCh, K. A. 2015 Cavitation nuclei in water exposed to transient pressures. J. Fluid Mech. 771, 424-448.

Atchley, A. A. \& Prosperetti, A. 1989 The crevice model of bubble nucleation. J. Acoust. Soc. Am. 86 (3), 1065-1084.

BANKOFF, S. G. 1958 Entrapment of gas in the spreading of a liquid over a rough surface. AIChE J. 4 (1), 24-26.

Benson, S. W. \& GerJuoy, E. 1949 The tensile strengths of liquids. I. Thermodynamic considerations. J. Chem. Phys. 17 (10), 914-918.

Blake, F. G. 1949 The Onset of Cavitation in Liquids. Tech. Memo. No. 12. Acoustics Res. Lab., Harvard University.

Borkent, B. M., Gekle, S., Prosperetti, A. \& Lohse, D. 2009 Nucleation threshold and deactivation mechanisms of nanoscopic cavitation nuclei. Phys. Fluids 21, 102003. 
Bremond, N., Arora, M., Ohl, C.-D. \& Lohse, D. 2005 Cavitation on surfaces. J. Phys.: Condens. Matter 17 (45), S3603-S3608.

Brennen, C. E. 1995 Cavitation and Bubble Dynamics. Oxford University Press.

BRENNEN, C. E. 2015 Cavitation in medicine. Interface Focus 5.

Briggs, L. J. 1950 Limiting negative pressure of water. J. Appl. Phys. 21, 721-722.

Chahine, G. L. 2015 Modeling cavitation dynamics: from nuclei to developed cavitation and erosion. Key Note Lecture of CAV 2015: 9th International Symposium on Cavitation, Lausanne, Switzerland.

Chen, D., Pan, L.-M. \& Ren, S. 2012 Prediction of bubble detachment diameter in flow boiling based on force analysis. Nucl. Engng Des. 243, 263-271.

Clift, R., Grace, J. R. \& Weber, M. E. 1978 Bubbles, Drops, and Particles. Academic.

Duhar, G. \& Colin, C. 2006 Dynamics of bubble growth and detachment in a viscous shear flow. Phys. Fluids 18, 077101.

Duineveld, P. C. 1995 The rise velocity and shape of bubbles in pure water at high Reynolds number. J. Fluid Mech. 292, 325-332.

Edwards, V. \& Newman, J. 1985 The asymmetric Graetz problem in channel flow. Intl J. Heat. Mass Transfer 28 (2), 503-505.

Epstein, P. S. \& Plesset, M. S. 1950 On the stability of gas bubbles in liquid-gas solutions. J. Chem. Phys. 18, 1505-1509.

Fernandez Rivas, D., Prosperetti, A., Zijlstra, A., Lohse, D. \& Gardeniers, H. J. G. E. 2010 Efficient sonochemistry through microbubbles generated with micromachined surfaces. Angew. Chem. 122 (50), 9893-9895.

FICK, A. 1855 Über diffusion. Ann. Phys. 170 (1), 59-86.

Fox, F. E. \& HeRZFELD, K. F. 1954 Gas bubbles with organic skin as cavitation nuclei. J. Acoust. Soc. Am. 26 (6), 984-989.

Franc, J.-P. \& Michel, J.-M. 2004 Fundamentals of Cavitation. Springer.

FritZ, W. 1935 Berechnung des maximalvolumens von dampfblasen. Physik. Z. 36, 379-384.

Groß, T. F., Ludwig, G. \& Pelz, P. F. 2015 Experimental evidence of nucleation from wall-bounded nuclei in a laminar flow. In CAV 2015: 9th International Symposium on Cavitation, 6-10 December 2015, Lausanne, Switzerland, J. Phys.: Conf. Series 656.

Groß, T. F., Ludwig, G. \& Pelz, P. F. 2016 Experimental and theoretical investigation of nucleation from wall-bounded nuclei in a laminar flow. In Proceedings of the 16th International Symposium on Transport Phenomena and Dynamics of Rotating Machinery, Honolulu, USA.

Guennoun, F., Farhat, M., Bouziad, Y. Ait \& Avellan, F. 2003 Experimental investigations of a particular travelling bubble cavitation. In CAV 2003: 5th International Symposium on Cavitation, 1-5 November 2003, Osaka, Japan.

Guennoun, M. F. 2006 Étude physique de l'apparition et du développement de la cavitation sur une aube isolée. PhD-Thesis Lausanne. EPFL.

Guzman, D. N., Hie, Y., Chen, S., Rivas, D. F., Sun, C., Lohse, D. \& Ahlers, G. 2016 Heat-flux enhancement by vapour-bubble nucleation in Rayleigh-Bénard turbulence. J. Fluid Mech. 787, 331-366.

Harvey, E. N., Barnes, D. K., Mcelroy, W. D., Whiteley, A. H., Pease, D. C. \& Cooper, K. W. 1944 Bubble formation in animals. I. Physical factors. J. Cell Physiol. 24 (1), 1-22.

HENRY, W. 1803 Experiments on the quantity of gases absorbed by water at different temperatures and under different pressures. Phil. Trans. R. Soc. Lond. 93, 29-274.

Higbie, R. 1935 The rate of absorption of a pure gas into a still liquid during short periods of exposure. Trans. AIChE 365-389.

Hsiao, C.-T., MA, J. \& Chahine, G. L. 2014 Multi-scale two-phase flow modeling of sheet and cloud cavitation. In 30th Symposium on Naval Hydrodynamics, 2-7 November 2014, Hobart, Australia.

Jones, S. F., Evans, G. M. \& Galvin, K. P. 1999 Bubble nucleation from gas cavities - a review. Adv. Colloid Interface Sci. 80, 27-50.

Jones, S. F., Galvin, K. P., Evans, G. M.\& Jameson, G. J. 1997 Carbonated water: the physics of the cycle of bubble production. Chem. Engng Sci. 53 (1), 169-173. 
Klausner, J. F., Mei, R., Bernhard, D. M. \& Zeng, L. Z. 1993 Vapor bubble departure in forced convection boiling. Intl J. Heat Mass Transfer 36 (3), 651-662.

Knapp, R. T., Daily, J. W. \& Hammitt, F. G. 1970 Cavitation. McGraw-Hill Book Company.

Liger-Belair, G. 2005 The physics and chemistry behind the bubbling properties of champagne and sparkling wines: a state-of-the-art review. J. Agric. Food Chem. 53 (8), 2788-2802.

Lochiel, A. C. \& CAlderbank, P. H. 1964 Mass transfer in the continuous phase around axisymmetric bodies of revolution. Chem. Engng Sci. 19, 471-484.

Lohse, D. \& Zhang, X. 2015 Surface nanobubbles and nanodroplets. Rev. Mod. Phys. 87, 981-1035.

Lubetkin, S. D. 1989 The nucleation and detachment of bubbles. J. Chem. Soc. Faraday Trans. 85, $1753-1764$.

Lubetkin, S. D. 1994 Bubble nucleation and growth. In Controlled Particle, Droplet and Bubble Formation (ed. D. J. Wedlock), pp. 159-187. Butterworth-Heinemann.

Ma, J., Hsiao, C.-T. \& Chahine, G. L. 2015 Modelling cavitating flows using an EulerianLagrangian approach and a nucleation model. In CAV 2015: 9th International Symposium on Cavitation, 6-10 December 2015, Lausanne, Switzerland, J. Phys.: Conf. Series 656.

Mørсh, K. A. 2007 Reflections on cavitation nuclei in water. Phys. Fluids 19, 072104.

NAhra, H. K. \& Kamonati, Y. 2003 Prediction of bubble diameter at detachment from a wall orifice in liquid cross-flow under reduced and normal gravity conditions. Chem. Engng Sci. 58, 55-69.

Parkin, B. R. \& Kermeen, R. W. 1963 The roles of convective air diffusion and liquid tensile stresses during cavitation inception. In Proceedings of IAHR Symposium on Cavitation and Hydraulic Machinery, Sendai, Japan.

Pelz, P. F., KeIL, T. \& Groß, T. F. 2017 The transition from sheet to cloud cavitation. J. Fluid Mech. 817, 439-454.

Pelz, P. F., Keil, T. \& Ludwig, G. 2014 Advanced Experimental and Numerical Techniques for Cavitation Erosion Prediction, chap. 9, Springer.

Peters, F. \& Els, C. 2012 An experimental study on slow and fast bubbles in tap water. Chem. Engng Sci. 82, 194-199.

Peters, F. \& HonZA, R. 2014 A benchmark experiment on gas cavitation. Exp. Fluids 55, 1786.

Prosperetti, A. 2017 Vapor bubbles. Annu. Rev. Fluid Mech. 49, 221-248.

RaYleigh, Lord 1879 On the capillary phenomena of jets. Proc. R. Soc. Lond. 29, 71-97.

VAn Rijsbergen, M. X. \& VAN Terwisga, T. J. C. 2011 High-speed micro-scale observations of nuclei-induced sheet cavitation. In WIMRC 3rd International Cavitation Forum 2011, Coventry, $U K$.

SARC, A., Oder, M. \& Dular, M. 2016 Can rapid pressure decrease induced by supercavitation efficiently eradicate Legionella Pneumophila bacteria? Desalin. Water Treat. 57 (5), 2184-2194.

Schlichting, H. \& Gersten, K. 2006 Grenzschichttheorie. Springer.

SCHNERR, G. H. \& SAUER., J. 2001 Physical and numerical modeling of unsteady cavitation dynamics. In Fourth International Conference on Multiphase Flow, New Orleans, USA.

Singhal, A. K., Li, H. Y., Athavale, M. M. \& Jiang, Y. 2001 Mathematical basis and validation of the full cavitation model. In ASME FEDSM'01, New Orleans, USA.

SPIRIDonov, E. K. 2015 Characteristics and calculation of cavitation mixers. Procedia Engng 129, $446-450$.

VerhaAgen, B. \& Fernandez Rivas, D. 2015 Measuring cavitation and its cleaning effect. Ultrason. Sonochem. 29, 619-628.

VAN WIJNGAARDEN, L. 1967 On the growth of small cavitation bubbles by convective diffusion. Intl J. Heat Mass Transfer 10 (2), 127-134.

Yount, D. E., Gillary, E. W. \& Hoffman, D. C. 1984 A microscopic investigation of bubble formation nuclei. J. Acoust. Soc. Am. 76 (5), 1511-1521.

Zijlstra, A., Fernandez Rivas, D., Gardeniers, H. J. G. E., Versluis, M. \& Lohse, D. 2015 Enhancing acoustic cavitation using artificial crevice bubbles. Ultrasonics 16, 512-523. 